This document is confidential and is proprietary to the American Chemical Society and its authors. Do not copy or disclose without written permission. If you have received this item in error, notify the sender and delete all copies.

\title{
Dithio- and Diselenophosphinate Thorium(IV) and Uranium(IV) Complexes: Molecular and Electronic Structure, Spectroscopy, and Transmetalation Reactivity
}

\begin{tabular}{|r|l|}
\hline Journal: & Inorganic Chemistry \\
\hline Manuscript ID & ic-2015-01342d.R2 \\
\hline Manuscript Type: & Article \\
\hline Date Submitted by the Author: & $10-$ Nov-2015 \\
\hline Complete List of Authors: & $\begin{array}{l}\text { Behrle, Andrew; University of Missouri, Chemistry } \\
\text { Kerridge, Andrew; Lancaster University, Chemistry } \\
\text { Walensky, Justin; University of Missouri, Columbia, Chemistry }\end{array}$ \\
\hline
\end{tabular}




\title{
Dithio- and Diselenophosphinate Thorium(IV) and Uranium(IV) Complexes: Molecular and Electronic Structure, Spectroscopy, and Transmetalation Reactivity
}

\author{
Andrew C. Behrle, ${ }^{1}$ Andrew Kerridge, ${ }^{2 *}$ and Justin R. Walensky ${ }^{1 *}$ \\ ${ }^{1}$ Department of Chemistry, University of Missouri, Columbia, MO 65211-7600 \\ ${ }^{2}$ Department of Chemistry, Lancaster University, Lancaster LA1 4YB, UK
}

\begin{abstract}
We report a comparison of the molecular and electronic structure of dithio- and diselenophosphinate, $\left(\mathrm{E}_{2} \mathrm{PR}_{2}\right)^{1-}, \mathrm{E}=\mathrm{S}, \mathrm{Se} ; \mathrm{R}={ }^{i} \mathrm{Pr},{ }^{t} \mathrm{Bu}$, with thorium(IV) and uranium(IV) complexes. For the thorium dithiophosphinate complexes, reaction of $\mathrm{ThCl}_{4}(\mathrm{DME})_{2}$ with four equivalents of $\mathrm{KS}_{2} \mathrm{PR}_{2}\left(\mathrm{R}={ }^{i} \mathrm{Pr},{ }^{t} \mathrm{Bu}\right)$ produced the homoleptic complexes, $\operatorname{Th}\left(\mathrm{S}_{2} \mathrm{P}^{i} \mathrm{Pr}_{2}\right)_{4}$, 1S-Th${ }^{i} \mathbf{P r}$, and $\mathrm{Th}\left(\mathrm{S}_{2} \mathrm{P}^{t} \mathrm{Bu}_{2}\right)_{4}$, , 2S-Th- $^{t} \mathbf{B u}$. The diselenophosphinate complexes were synthesized in a similar manner using $\mathrm{KSe}_{2} \mathrm{PR}_{2}$ to produce $\mathrm{Th}\left(\mathrm{Se}_{2} \mathrm{P}^{i} \mathrm{Pr}_{2}\right)_{4}$, $\mathbf{1 S e}-\mathbf{T h}-{ }^{i} \mathbf{P r}$, and $\mathrm{Th}\left(\mathrm{Se}_{2} \mathrm{P}^{t} \mathrm{Bu}_{2}\right)_{4}$, , 2Se-
\end{abstract} Th- ${ }^{t} \mathbf{B u} . \quad \mathrm{U}\left(\mathrm{S}_{2} \mathrm{P}^{i} \mathrm{Pr}_{2}\right)_{4}, \mathbf{1 S}-\mathbf{U}-{ }^{i} \mathbf{P r}$, could be made directly from $\mathrm{UCl}_{4}$ and four equivalents of $\mathrm{KS}_{2} \mathrm{P}^{i} \operatorname{Pr}_{2}$. With $\left(\mathrm{Se}_{2} \mathrm{P}^{i} \mathrm{Pr}_{2}\right)^{1-}$, using $\mathrm{UCl}_{4}$ and three or four equivalents of $\mathrm{KSe}_{2} \mathrm{P}^{i} \operatorname{Pr}_{2}$ yielded the mono-chloride product $\mathrm{U}\left(\mathrm{Se}_{2} \mathrm{P}^{i} \mathrm{Pr}_{2}\right)_{3} \mathrm{Cl}, \mathbf{3 S e}-\mathbf{U}^{i \mathbf{P r}}-\mathbf{C l}$, but using $\mathrm{UI}_{4}(1,4 \text {-dioxane })_{2}$ produced the homoleptic $\mathrm{U}\left(\mathrm{Se}_{2} \mathrm{P}^{i} \mathrm{Pr}_{2}\right)_{4}$, 1Se-U- ${ }^{i} \mathbf{P r}$. Similarly, the reaction of $\mathrm{UCl}_{4}$ with four equivalents of $\mathrm{KS}_{2} \mathrm{P}^{t} \mathrm{Bu}_{2}$ yielded $\mathrm{U}\left(\mathrm{S}_{2} \mathrm{P}^{t} \mathrm{Bu}_{2}\right)_{4}, \mathbf{2 S}-\mathbf{U}_{-}{ }^{t} \mathbf{B u}$, while the reaction with $\mathrm{KSe}_{2} \mathrm{P}^{t} \mathrm{Bu}_{2}$ resulted in the formation of $\mathrm{U}\left(\mathrm{Se}_{2} \mathrm{P}^{t} \mathrm{Bu}_{2}\right)_{3} \mathrm{Cl}, 4 \mathbf{S e}-\mathbf{U}^{\mathbf{t B u}}-\mathbf{C l}$. Using $\mathrm{UI}_{4}(1,4 \text {-dioxane })_{2}$ and four equivalents of $\mathrm{KSe}_{2} \mathrm{P}^{t} \mathrm{Bu}_{2}$ with $\mathrm{UCl}_{4}$ in acetonitrile yielded $\mathrm{U}\left(\mathrm{Se}_{2} \mathrm{P}^{t} \mathrm{Bu}_{2}\right)_{4}, \mathbf{2 S e - U}-{ }^{t} \mathbf{B u}$. Transmetalation reactions

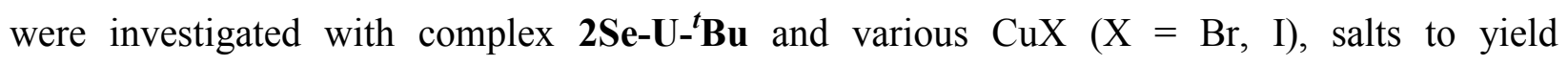
$\mathrm{U}\left(\mathrm{Se}_{2} \mathrm{P}^{t} \mathrm{Bu}_{2}\right)_{3} \mathrm{X},\left(\mathbf{6 S e}-\mathbf{U}^{\mathrm{tBu}}-\mathbf{B r}\right.$, and $\left.7 \mathrm{Se}-\mathbf{U}^{\mathrm{tBu}}-\mathbf{I}\right)$ and 0.25 equivalents of $\left[\mathrm{Cu}\left(\mathrm{Se}_{2} \mathrm{P}^{t} \mathrm{Bu}_{2}\right)\right]_{4}, \mathbf{8 S e}-$ $\mathbf{C u}-{ }^{t} \mathbf{B u}$. Additionally $\mathbf{2 S e}-\mathbf{U}_{-}{ }^{t} \mathbf{B u}$ underwent transmetalation reactions with $\mathrm{Hg}_{2} \mathrm{~F}_{2}$ and $\mathrm{ZnCl}_{2}$ to yield $\mathrm{U}\left(\mathrm{Se}_{2} \mathrm{P}^{t} \mathrm{Bu}_{2}\right)_{3} \mathrm{~F}, \mathbf{6}$, and $\mathrm{U}\left(\mathrm{Se}_{2} \mathrm{P}^{t} \mathrm{Bu}_{2}\right)_{3} \mathrm{Cl}, \mathbf{4 S e}-\mathbf{U}^{\mathrm{tBu}}-\mathbf{C l}$, respectively. The molecular structures were analyzed using ${ }^{1} \mathrm{H},{ }^{13} \mathrm{C},{ }^{31} \mathrm{P}$, and ${ }^{77} \mathrm{Se}$ NMR and IR spectroscopy and structurally 
characterized using X-ray crystallography. Using the QTAIM approach, the electronic structure of all homoleptic complexes were probed and show slightly more covalent bonding character in actinide-selenium bonds over actinide-sulfur bonds.

\section{Introduction}

Recycling of spent nuclear fuel is important if nuclear energy is to be a viable source for a portion of the world's increasing energy requirements. In recent years, extractors bearing soft donor atoms have been shown to have selectivity for actinides over lanthanides. ${ }^{1-3}$ In fact, Cyanex 301, a dithiophosphinic acid, is commercially used to extract late minor actinides from lanthanides. ${ }^{4}$ This is presumed to be due to actinide-ligand bonding having more covalent character than lanthanides but further study is warranted. In this regard, the synthesis of dithioand diselenophosphinate complexes with alkyl-substituents provides a platform from which to investigate this phenomenon.

The Gaunt group recently reported a series of $4 \mathrm{f}$ and $5 \mathrm{f}$ diphenyldiselenophosphinate complexes which showed enhanced covalent character in the actinide series than their lanthanide counterparts. ${ }^{5}$ We followed with a comparison of sulfur- and selenium-based phosphonate compounds which observed actinide-selenium bonds had higher covalency than actinide-sulfur bonds. ${ }^{6}$ For strongly oxophilic, Lewis acidic metal centers such as the actinides, this was an unexpected result and opens up new opportunities on how to describe actinide-ligand bonding.

The reactivity of actinide complexes with soft donor ligands is limited. Insertion of $\mathrm{CO}_{2}$ and $\mathrm{CS}_{2}$ into uranium-thiolato, ${ }^{7,8}$-sulfide, -selenide-, and telluride ${ }^{9}$ bonds has been reported. In our previous work we noted that insertion reactivity was not observed with dithio- and diselenophosphonate complexes but realized this could be due to steric crowding around the metal center. Given our interest in coinage metal chemistry, ${ }^{10}$ which have a propensity for soft 
donor atoms such as sulfur and selenium, transmetalation reactions with soft metal salts seemed plausible. These reactions are advantageous to achieve stoichiometric substitution at one site on the actinide and hence examine structure, bonding, and spectroscopic differences. Herein, we report the synthesis of $\mathrm{An}\left(\mathrm{E}_{2} \mathrm{PR}_{2}\right)_{4}\left(\mathrm{An}=\mathrm{Th}, \mathrm{U} ; \mathrm{E}=\mathrm{S}, \mathrm{Se} ; \mathrm{R}={ }^{i} \mathrm{Pr},{ }^{t} \mathrm{Bu}\right)$ complexes and transmetalation reactions with $\mathrm{U}\left(\mathrm{Se}_{2} \mathrm{P}^{t} \mathrm{Bu}_{2}\right)_{4}$ and $\mathrm{CuBr}, \mathrm{CuI}, \mathrm{ZnCl}_{2}$, and $\mathrm{Hg}_{2} \mathrm{~F}_{2}$ salts to produce compounds of the form $\mathrm{XU}\left(\mathrm{Se}_{2} \mathrm{P}^{t} \mathrm{Bu}_{2}\right)_{3}$.

\section{Experimental}

General considerations. The syntheses and manipulations described below were conducted using standard Schlenk and glovebox techniques. All reactions were conducted in a Vacuum Atmospheres inert atmosphere $\left(\mathrm{N}_{2}\right)$ glovebox. THF, toluene, and hexanes were purchased anhydrous, stored over activated $4 \AA$ molecular sieves, and sparged with nitrogen prior to use. Methylene chloride and ethanol (200 proof) were dried over activated $4 \AA$ molecular sieves and sparged with nitrogen for thirty minutes prior to use. $\left[\mathrm{ThCl}_{4}(\mathrm{DME})_{2}\right],{ }^{11}$ and $\left[\mathrm{UCl}_{4}\right]^{12}$ were synthesized as previously described. $\left[\mathrm{Th}\left(\mathrm{S}_{2} \mathrm{P}^{i} \mathrm{Pr}_{2}\right)_{4}\right]^{13}$ and $\left[\mathrm{KS}_{2} \mathrm{P}^{i} \mathrm{Pr}_{2}\right]$, ${ }^{14}$ were synthesized using modified literature procedures (vide infra). Diisopropylphosphine (10\% in hexanes), di-tertbutylphosphine, sublimed sulfur, selenium, potassium hydroxide, $\left[\mathrm{Cu}(\mathrm{NCMe})_{4}\right] \mathrm{PF}_{6}, \operatorname{copper}(\mathrm{I})$ chloride, $\mathrm{Hg}_{2} \mathrm{~F}_{2}, \mathrm{HgCl}_{2}$, copper(I) bromide, and copper(I) iodide were purchased from commercial suppliers and used without further purification. Benzene- $d_{6}$ and THF- $d_{8}$ (Cambridge Isotope Laboratories) were dried over molecular sieves and degassed with three freeze-evacuatethaw cycles. $\mathrm{D}_{2} \mathrm{O}$ (Cambridge Isotope Laboratories) was used as received. All ${ }^{1} \mathrm{H},{ }^{13} \mathrm{C}$, ${ }^{31} \mathrm{P}$, and ${ }^{77} \mathrm{Se}$ NMR data were obtained on a $300 \mathrm{MHz}$ DRX or $500 \mathrm{MHz}$ DRX Bruker spectrometer. ${ }^{1} \mathrm{H}$ NMR shifts given were referenced internally to the residual solvent peaks at $\delta 7.16 \mathrm{ppm}$ $\left(\mathrm{C}_{6} \mathrm{D}_{5} \mathrm{H}\right), \delta 1.72 \mathrm{ppm}\left(\mathrm{C}_{4} \mathrm{D}_{7} \mathrm{HO}\right), \delta 4.79$ (HDO). ${ }^{13} \mathrm{C}$ NMR shifts given were referenced internally 
to the residual peaks at $\delta 128.0 \mathrm{ppm}\left(\mathrm{C}_{6} \mathrm{D}_{6}\right)$ or $\delta 67.20\left(\mathrm{C}_{4} \mathrm{D}_{8} \mathrm{O}\right) .{ }^{31} \mathrm{P}$ NMR spectra were externally referenced to $0.00 \mathrm{ppm}$ with $5 \% \mathrm{H}_{3} \mathrm{PO}_{4}$ in $\mathrm{D}_{2} \mathrm{O} .{ }^{77} \mathrm{Se} \mathrm{NMR}$ shifts given were referenced externally to 460.00 ppm with diphenyl diselenide in $\mathrm{C}_{6} \mathrm{D}_{6}, \mathrm{C}_{4} \mathrm{D}_{8} \mathrm{O}$, or $\mathrm{D}_{2} \mathrm{O}$. Infrared spectra were recorded as $\mathrm{KBr}$ pellets on Perkin-Elmer Spectrum One FT-IR spectrometer. Elemental analyses were performed by Atlantic Microlab, Inc. (Norcross, GA).

Crystallographic Data Collection and Structure Determination. The selected single crystal was mounted on nylon cryoloops using viscous hydrocarbon oil. X-ray data collection was performed at 173(2) or 100(2) K. The X-ray data were collected on a Bruker CCD diffractometer with monochromated Mo-K $\alpha$ radiation $(\lambda=0.71073 \AA)$ or $\mathrm{Cu}-\mathrm{K} \alpha$ radiation $(\lambda=1.54178 \AA)$. The data collection and processing utilized Bruker Apex 2 suite of programs. ${ }^{15}$ The structures were solved using direct methods and refined by full-matrix least-squares methods on F2 using Bruker SHELX-2014/7 program. $^{16}$ All non-hydrogen atoms were refined with anisotropic displacement parameters. All hydrogen atoms were placed at calculated positions and included in the refinement using a riding model. Thermal ellipsoid plots were prepared by using X-seed ${ }^{17}$ with $50 \%$ of probability displacements for non-hydrogen atoms. Crystal data and details for data collection for complexes 1-5, 7-10, 13, and 15 are provided in Tables 1 and 2. Significant bond distances and angles are listed in Tables 5-9 and 10.

$\mathbf{K S}_{\mathbf{2}} \mathbf{P}^{i} \mathbf{P r}_{\mathbf{2}}$. An oven-dried $100 \mathrm{~mL}$ Schlenk flask was charged with diisopropylphosphine (10 g, $8.46 \mathrm{mmol}$ ) and cycled onto a Schlenk line. $30 \mathrm{~mL}$ of 200 proof ethanol was added to diisopropylphosphine followed by potassium hydroxide $(475 \mathrm{mg}, 8.46 \mathrm{mmol})$ and elemental sulfur (543 mg, $2.12 \mathrm{mmol})$. The reaction was allowed to stir at room temperature for $12 \mathrm{~h}$ to yield a colorless solution. The ethanol was removed under vacuum to yield a white precipitate and the Schlenk flask was taken inside the glove box. The white precipitate was washed twice 
with $20 \mathrm{~mL}$ of diethyl ether, filtered over a frit, and dried to yield a white powder $(1.77 \mathrm{~g}, 95 \%)$. ${ }^{1} \mathrm{H}$ NMR $\left(\mathrm{D}_{2} \mathrm{O}, 25{ }^{\circ} \mathrm{C}\right): \delta 2.22-2.16\left(\mathrm{~m}, 2 \mathrm{H}, \mathrm{CH}\left(\mathrm{CH}_{3}\right)\right), 1.18\left(\mathrm{dd},{ }^{3} J_{\mathrm{P}-\mathrm{H}}=18.0 \mathrm{~Hz},{ }^{3} J_{\mathrm{H}-\mathrm{H}}=7.0 \mathrm{~Hz}\right.$, $\left.12 \mathrm{H}, \mathrm{CH}\left(\mathrm{CH}_{3}\right)\right) .{ }^{13} \mathrm{C}\left\{{ }^{1} \mathrm{H}\right\}\left(\mathrm{D}_{2} \mathrm{O}, 25{ }^{\circ} \mathrm{C}\right.$, uncorrected $): \delta 33.03\left(\mathrm{~d},{ }^{1} J_{\mathrm{P}-\mathrm{C}}=50.3 \mathrm{~Hz}\right), 15.74 .{ }^{31} \mathrm{P}\left\{{ }^{1} \mathrm{H}\right\}$ $\left(\mathrm{D}_{2} \mathrm{O}, 25^{\circ} \mathrm{C}\right): \delta 95.6 . \mathrm{IR}\left(\mathrm{cm}^{-1}\right): 2982(\mathrm{~s}), 2870(\mathrm{~s}), 1479(\mathrm{~m}), 1388(\mathrm{~m}), 1365(\mathrm{~m}), 1177(\mathrm{~s}), 1124$ (s), $1110(\mathrm{~s}), 1025(\mathrm{~s}), 804(\mathrm{~m}), 625(\mathrm{~s}), 612(\mathrm{~s}), 520(\mathrm{~m}), 473(\mathrm{~m})$.

$\mathbf{K S}_{\mathbf{2}} \mathbf{P}^{t} \mathbf{B u}_{2}$. An oven-dried $120 \mathrm{~mL}$ Schlenk flask was charged with ${ }^{t} \mathrm{Bu}{ }_{2} \mathrm{PH}(1.0 \mathrm{~g}, 6.84 \mathrm{mmol})$ followed by THF ( $3 \mathrm{~mL}$ ). The Schlenk flask was cycled onto the Schlenk line and $20 \mathrm{~mL}$ of 200 proof ethanol was added followed by potassium hydroxide $(384 \mathrm{mg}, 6.84 \mathrm{mmol})$. The mixture was stirred until the potassium hydroxide was dissolved followed by addition of element sulfur (439 $\mathrm{mg}, 1.71 \mathrm{mmol}$ ). The reaction was stirred until the sulfur was consumed. The solvent was removed under vacuum to yield a white solid which was triturated with $20 \mathrm{~mL}$ of hexanes to yield a white microcrystalline powder $(1.46 \mathrm{~g}, 86 \%) .{ }^{1} \mathrm{H} \mathrm{NMR}\left(\mathrm{D}_{2} \mathrm{O}, 25{ }^{\circ} \mathrm{C}\right): \delta 1.37\left(\mathrm{~d},{ }^{3} J_{\mathrm{P}-\mathrm{H}}=16\right.$ $\left.\mathrm{Hz}, 18 \mathrm{H}, \mathrm{C}\left(\mathrm{CH}_{3}\right)_{3}\right) .{ }^{13} \mathrm{C}\left\{{ }^{1} \mathrm{H}\right\}\left(\mathrm{D}_{2} \mathrm{O}, 25{ }^{\circ} \mathrm{C}\right.$, uncorrected $): \delta 40.97\left(\mathrm{~d},{ }^{1} J_{\mathrm{P}-\mathrm{C}}=41 \mathrm{~Hz}\right), 27.40\left(\mathrm{~d},{ }^{2} J_{\mathrm{P}-}\right.$ $\mathrm{C}=2.0 \mathrm{~Hz}) \cdot{ }^{31} \mathrm{P}\left\{{ }^{1} \mathrm{H}\right\}\left(\mathrm{D}_{2} \mathrm{O}, 25{ }^{\circ} \mathrm{C}\right): \delta$ 112.4. IR $\left(\mathrm{cm}^{-1}\right): 2963(\mathrm{~s}), 2891(\mathrm{~s}), 1473(\mathrm{~m}), 1383(\mathrm{~m})$, $1358(\mathrm{~m}), 1173(\mathrm{~s}), 1127$ (s), 1108 (s), $1016(\mathrm{~s}), 806$ (m), 629 (s), 609 (s), 517 (m), 469 (m).

$\mathbf{K S e}_{2} \mathbf{P}^{i} \mathbf{P r}_{2}$. Following the same procedure for $\mathrm{KS}_{2} \mathrm{P}^{i} \mathrm{Pr}_{2}$, diisopropylphosphine $(10 \mathrm{~g}, 8.46$ mmol), potassium hydroxide (475 $\mathrm{mg}, 8.46 \mathrm{mmol})$, and elemental selenium (1.34 g, $17.0 \mathrm{mmol})$ to yield a white powder $(2.65 \mathrm{~g}, 99 \%) .{ }^{1} \mathrm{H} \mathrm{NMR}\left(\mathrm{D}_{2} \mathrm{O}, 25^{\circ} \mathrm{C}\right): \delta 2.26-2.20\left(\mathrm{~m}, 2 \mathrm{H}, \mathrm{CH}\left(\mathrm{CH}_{3}\right)\right)$, $1.18\left(\mathrm{dd},{ }^{3} J_{\mathrm{P}-\mathrm{H}}=20.0 \mathrm{~Hz},{ }^{3} J_{\mathrm{H}-\mathrm{H}}=7.0 \mathrm{~Hz}, 12 \mathrm{H}, \mathrm{CH}\left(\mathrm{CH}_{3}\right)\right) .{ }^{13} \mathrm{C}\left\{{ }^{1} \mathrm{H}\right\}\left(\mathrm{D}_{2} \mathrm{O}, 25{ }^{\circ} \mathrm{C}\right.$, uncorrected $): \delta$ $32.93\left(\mathrm{~d},{ }^{1} J_{\mathrm{P}-\mathrm{C}}=36.0 \mathrm{~Hz}\right), 16.58\left(\mathrm{~d},{ }^{2} J_{\mathrm{P}-\mathrm{C}}=13.0 \mathrm{~Hz}\right) \cdot{ }^{31} \mathrm{P}\left\{{ }^{1} \mathrm{H}\right\}\left(\mathrm{D}_{2} \mathrm{O}, 25{ }^{\circ} \mathrm{C}\right): \delta 70.1(\mathrm{~s}+\mathrm{d}$ satellites, $\left.{ }^{1} J_{\mathrm{Se}-\mathrm{P}}=553.0 \mathrm{~Hz}\right) .{ }^{77} \mathrm{Se}\left\{{ }^{1} \mathrm{H}\right\}\left(\mathrm{D}_{2} \mathrm{O}, 25{ }^{\circ} \mathrm{C}\right): \delta-192.01 \quad\left(\mathrm{~d},{ }^{1} J_{\mathrm{P}-\mathrm{Se}}=553 \mathrm{~Hz}\right) . \quad \mathrm{IR}\left(\mathrm{cm}^{-1}\right)$ : 2970 (s), 2913 (s), 2862 (s), 1459 (s), 1379 (s), 1291 (m), 1236 (m), 1160 (w), 1100 (m), 1083 (w), $1021(\mathrm{~s}), 965(\mathrm{w}), 929(\mathrm{w}), 883(\mathrm{~s}), 837(\mathrm{w}), 655(\mathrm{~s}), 593(\mathrm{~s})$. 
$\mathbf{K S e}_{2} \mathbf{P}^{t} \mathbf{B u}_{2}$. A $120 \mathrm{~mL}$ Schlenk flask was charged with ${ }^{t} \mathrm{Bu}_{2} \mathrm{PH}(1.0 \mathrm{~g}, 6.84 \mathrm{mmol})$ and THF $(3 \mathrm{~mL})$. The flask was cycled onto a Schlenk line and $20 \mathrm{~mL}$ of 200 proof ethanol was added followed by potassium hydroxide $(384 \mathrm{mg}, 6.84 \mathrm{mmol})$. The mixture was stirred until the potassium hydroxide was dissolved followed by addition of elemental selenium (1.08 $\mathrm{g}, 13.7$ mmol). The reaction was stirred overnight and filtered via cannula. The solvent was removed under vacuum to yield a yellow solid which was washed with $20 \mathrm{~mL}$ of hexanes, filtered over a frit, and dried under vacuum to yield a white microcrystalline powder $(1.74 \mathrm{~g}, 74 \%)$. ${ }^{1} \mathrm{H}$ NMR $\left(\mathrm{D}_{2} \mathrm{O}, 25^{\circ} \mathrm{C}\right): \delta 1.42\left(\mathrm{~d},{ }^{3} \mathrm{~J}_{\mathrm{P}-\mathrm{H}}=17.0 \mathrm{~Hz}, 18 \mathrm{H}, \mathrm{C}\left(\mathrm{CH}_{3}\right)_{3}\right) .{ }^{13} \mathrm{C}\left\{{ }^{1} \mathrm{H}\right\}\left(\mathrm{D}_{2} \mathrm{O}, 25{ }^{\circ} \mathrm{C}\right.$, uncorrected $): \delta$ $40.27\left(\mathrm{~d},{ }^{1} J_{\mathrm{P}-\mathrm{C}}=25 \mathrm{~Hz}\right), 27.79\left(\mathrm{~d},{ }^{2} J_{\mathrm{P}-\mathrm{C}}=2.5 \mathrm{~Hz}\right) .{ }^{31} \mathrm{P}\left\{{ }^{1} \mathrm{H}\right\}\left(\mathrm{D}_{2} \mathrm{O}, 25{ }^{\circ} \mathrm{C}\right): \delta 95.7(\mathrm{~s}+\mathrm{d}$ satellites, $\left.{ }^{1} J_{\mathrm{Se}-\mathrm{P}}=556 \mathrm{~Hz}\right) .{ }^{77} \mathrm{Se}\left\{{ }^{1} \mathrm{H}\right\}\left(\mathrm{D}_{2} \mathrm{O}, 25{ }^{\circ} \mathrm{C}\right): \delta-153.5\left(\mathrm{~d},{ }^{1} J_{\mathrm{P}-\mathrm{Se}}=556 \mathrm{~Hz}\right) . \mathrm{IR}\left(\mathrm{cm}^{-1}\right): 2962(\mathrm{~m}), 2911$ (m), 1459 (m), 1382 (m), 1357 (m), 1170 (s), 1088 (s), 1019 (s), 803 (m), 599 (w), 578 (w), 537 (s), $484(\mathrm{~m})$.

$\mathbf{T h}\left(\mathbf{S}_{2} \mathbf{P}^{i} \mathbf{P r}_{2}\right)_{4}$, $\mathbf{1 S - T h}-{ }^{i} \mathbf{P r}$. A $20 \mathrm{~mL}$ scintillation vial was charged with $\mathrm{ThCl}_{4}(\mathrm{DME})_{2}(174 \mathrm{mg}$, $0.314 \mathrm{mmol})$ and THF $(3 \mathrm{~mL})$. A second $20 \mathrm{~mL}$ scintillation vial was charged with $\mathrm{KS}_{2} \mathrm{P}^{i} \operatorname{Pr}_{2}$ (283 mg, $1.28 \mathrm{mmol}$ ) and THF (3 mL). The solution of $\mathrm{KS}_{2} \mathrm{P}^{i} \mathrm{Pr}_{2}$ was added to a stirring solution of $\mathrm{ThCl}_{4}(\mathrm{DME})_{2}$ and allowed to stir for $14 \mathrm{~h}$ at room temperature. The reaction solvent was removed under vacuum and the precipitate was extracted twice with toluene, filtered over a bed of Celite, concentrated, and layered with $\mathrm{Et}_{2} \mathrm{O}$ to yield a white microcrystalline material (180 $\mathrm{mg}, 60 \%$ ). Colorless X-ray quality crystals were grown from a concentrated $\mathrm{Tol} / \mathrm{Et}_{2} \mathrm{O}$ mixture at $-20{ }^{\circ} \mathrm{C} .{ }^{1} \mathrm{H}$ NMR $\left(\mathrm{C}_{6} \mathrm{D}_{6}, 25{ }^{\circ} \mathrm{C}\right): \delta 2.36\left(\mathrm{~d} \mathrm{sep},{ }^{2} J_{\mathrm{P}-\mathrm{H}}=10.0 \mathrm{~Hz},{ }^{3} J_{\mathrm{H}-\mathrm{H}}=7.5 \mathrm{~Hz}, 8 \mathrm{H}, \mathrm{CH}\left(\mathrm{CH}_{3}\right)_{2}\right)$, $1.23\left(\mathrm{dd},{ }^{3} J_{\mathrm{P}-\mathrm{H}}=20.0 \mathrm{~Hz},{ }^{3} J_{\mathrm{H}-\mathrm{H}}=7.5 \mathrm{~Hz}, \mathrm{CH}\left(\mathrm{CH}_{3}\right)_{2}\right) .{ }^{13} \mathrm{C}\left\{{ }^{1} \mathrm{H}\right\}\left(\mathrm{C}_{6} \mathrm{D}_{6}, 25{ }^{\circ} \mathrm{C}\right): \delta 35.19\left(\mathrm{~d},{ }^{1} J_{\mathrm{P}-\mathrm{C}}=\right.$ 43.0 Hz), 17.00. ${ }^{31} \mathrm{P}\left\{{ }^{1} \mathrm{H}\right\}\left(\mathrm{C}_{6} \mathrm{D}_{6}, 25{ }^{\circ} \mathrm{C}\right): \delta 84.9 . \mathrm{IR}\left(\mathrm{cm}^{-1}\right): 2960$ (s), 2925 (s), $2860(\mathrm{~s}), 2427(\mathrm{w})$, 1460 (s), 1387 (s), 1288 (m), 1245 (m), 1160 (w), 1090 (m), 1048 (m), 1026 (m), 930 (m), 881 
(s), 838 (w), 690 (m), 671 (s), 632 (s), 500 (s). Anal. calcd. for $\mathrm{C}_{24} \mathrm{H}_{56} \mathrm{P}_{4} \mathrm{~S}_{8} \mathrm{Th}$ : C, 30.12\%; H, 5.90\%. Found C, 29.90\%; H, 5.66\%.

$\mathbf{T h}\left(\mathbf{S}_{\mathbf{2}} \mathbf{P}^{\boldsymbol{t}} \mathbf{B u}_{2}\right)_{4}$, 2S-Th- ${ }^{t} \mathbf{B u}$. A $20 \mathrm{~mL}$ scintillation vial was charged with $\mathrm{ThCl}_{4}(\mathrm{DME})_{2}(109 \mathrm{mg}$, $0.196 \mathrm{mmol})$ and THF $(3 \mathrm{~mL})$. A second $20 \mathrm{~mL}$ scintillation vial was charged with $\mathrm{KS}_{2} \mathrm{P}^{t} \mathrm{Bu}_{2}$ (200 mg, $0.805 \mathrm{mmol})$ and THF $(3 \mathrm{~mL})$. The $\mathrm{KS}_{2} \mathrm{P}^{t} \mathrm{Bu}_{2}$ was added to a stirring solution of $\mathrm{ThCl}_{4}(\mathrm{DME})_{2}$ and allowed to stir for $14 \mathrm{~h}$ at room temperature. The solvent removed under vacuum, extracted with toluene, and filtered over Celite. The toluene was removed under vacuum to yield a pale yellow residue which was extracted with THF, filtered over Celite and concentrated to yield a white microcrystalline solid (84 mg, 42\%). X-ray quality crystals were grown from a concentrated THF/hexanes mixture at room temperature. ${ }^{1} \mathrm{H}$ NMR $\left(\mathrm{C}_{6} \mathrm{D}_{6}, 25{ }^{\circ} \mathrm{C}\right)$ : $\delta 1.43\left(\mathrm{~d},{ }^{3} J_{\mathrm{P}-\mathrm{H}}=15.0 \mathrm{~Hz}, 72 \mathrm{H}, \mathrm{C}\left(\mathrm{CH}_{3}\right)_{3}\right) .{ }^{13} \mathrm{C}\left\{{ }^{1} \mathrm{H}\right\}\left(\mathrm{C}_{6} \mathrm{D}_{6}, 25{ }^{\circ} \mathrm{C}\right): \delta 42.97\left(\mathrm{~d},{ }^{1} J_{\mathrm{P}-\mathrm{C}}=34.0 \mathrm{~Hz}\right)$, 27.44. ${ }^{31} \mathrm{P}\left\{{ }^{1} \mathrm{H}\right\}\left(\mathrm{C}_{6} \mathrm{D}_{6}, 25^{\circ} \mathrm{C}\right): \delta 99.1 . \mathrm{IR}\left(\mathrm{cm}^{-1}\right): 2990(\mathrm{~m}), 2964(\mathrm{~m}), 2904(\mathrm{~m}), 2868(\mathrm{~m}), 1475$ (s), $1390(\mathrm{~m}), 1364(\mathrm{~m}), 1181$ (s), 1082 (s), $1088(\mathrm{~s}), 939$ (m), 804 (m), 620 (s), 518 (m), 467 (m), 441 (m). Anal. calcd. for $\mathrm{C}_{32} \mathrm{H}_{72} \mathrm{P}_{4} \mathrm{~S}_{8} \mathrm{Th} \cdot 0.5(\mathrm{THF})$ : C, 36.94\%; H, 6.93\%. Found C, 36.99\%; $\mathrm{H}, 6.87 \%$.

$\mathbf{T h}\left(\mathbf{S e}_{2} \mathbf{P}^{i} \mathbf{P r}_{2}\right)_{4}$, 1Se-Th- ${ }^{i} \mathbf{P r}$. A $20 \mathrm{~mL}$ scintillation vial was charged with $\mathrm{ThCl}_{4}(\mathrm{DME})_{2}(97 \mathrm{mg}$, $0.175 \mathrm{mmol})$ and THF $(3 \mathrm{~mL}) \mathrm{A}$ second $20 \mathrm{~mL}$ scintillation vial was charged with $\mathrm{KSe}_{2} \mathrm{P}^{i} \operatorname{Pr}_{2}$ (225 mg, $0.716 \mathrm{mmol}$ ) and THF (3 mL). Both vials were placed inside a $-20{ }^{\circ} \mathrm{C}$ freezer for 10 min. The solution of $\mathrm{KSe}_{2} \mathrm{P}^{\mathrm{i}} \mathrm{Pr}_{2}$ was added to a stirring solution of $\mathrm{ThCl}_{4}(\mathrm{DME})_{2}$ and allowed to stir at room temperature for $14 \mathrm{~h}$ resulting in a yellow solution. The reaction solvent was removed under vacuum and the precipitate was extracted twice with toluene, filtered over a bed a Celite, and the solvent removed under reduced pressure to yield a white precipitate (182 $\mathrm{mg}$, $78 \%$ ). Colorless X-ray quality crystals were grown from concentrated $\mathrm{Tol} / \mathrm{Et}_{2} \mathrm{O}$ mixture at -23 
${ }^{\circ} \mathrm{C} .{ }^{1} \mathrm{H}$ NMR $\left(\mathrm{C}_{6} \mathrm{D}_{6}, 25{ }^{\circ} \mathrm{C}\right): \delta 2.41-2.39\left(\mathrm{~m}, 8 \mathrm{H}, \mathrm{CH}\left(\mathrm{CH}_{3}\right)_{2}\right), 1.25\left(\mathrm{dd},{ }^{3} J_{\mathrm{P}-\mathrm{H}}=20.0 \mathrm{~Hz},{ }^{3} J_{\mathrm{H}-\mathrm{H}}=\right.$ $\left.7.0 \mathrm{~Hz}, 48 \mathrm{H}, \mathrm{CH}\left(\mathrm{CH}_{3}\right)_{2}\right) .{ }^{13} \mathrm{C}\left\{{ }^{1} \mathrm{H}\right\}\left(\mathrm{C}_{6} \mathrm{D}_{6}, 25{ }^{\circ} \mathrm{C}\right): \delta 34.12\left(\mathrm{~d},{ }^{1} J_{\mathrm{P}-\mathrm{C}}=29.0 \mathrm{~Hz}\right), 17.69 .{ }^{31} \mathrm{P}\left\{{ }^{1} \mathrm{H}\right\}$ $\left(\mathrm{C}_{6} \mathrm{D}_{6}, 25^{\circ} \mathrm{C}\right): \delta 57.6\left(\mathrm{~s}+\mathrm{d}\right.$ satellites, $\left.{ }^{1} J_{\text {Se-P }}=500 \mathrm{~Hz}\right) .{ }^{77} \mathrm{Se}\left\{{ }^{1} \mathrm{H}\right\}\left(\mathrm{C}_{6} \mathrm{D}_{6}, 25{ }^{\circ} \mathrm{C}\right): \delta 6.4\left(\mathrm{~d},{ }^{1} J_{\mathrm{P}-\mathrm{Se}}=\right.$ $500 \mathrm{~Hz}) . \mathrm{IR}\left(\mathrm{cm}^{-1}\right): 2960$ (s), 2925 (m), 2867 (m), 1459 (s), 1384 (s), 1237 (m), 1159 (w), 1091 (w), $1047(w), 1023(w), 930(w), 878$ (m), 837 (w), 649 (s), 615 (m), 538 (s). 478 (m). Anal. calcd. for $\mathrm{C}_{24} \mathrm{H}_{56} \mathrm{P}_{4} \mathrm{Se}_{8}$ Th: C, 21.64\%; H, 4.24\%. Found C, 21.82\%; H, 4.13\%.

Th( $\left.\mathbf{S e}_{2} \mathbf{P}^{t} \mathbf{B u}_{2}\right)_{4}$, 2Se-Th- ${ }^{t} \mathbf{B u}$. A $20 \mathrm{~mL}$ scintillation vial was charged with $\mathrm{ThCl}_{4}(\mathrm{DME})_{2}(77 \mathrm{mg}$, $0.138 \mathrm{mmol})$ and THF $(3 \mathrm{~mL})$. A second $20 \mathrm{~mL}$ scintillation vial was charged with $\mathrm{KSe}_{2} \mathrm{P}^{t} \mathrm{Bu}_{2}$ (194 mg, $0.567 \mathrm{mmol}$ ) and THF $(3 \mathrm{~mL})$. The $\mathrm{KSe}_{2} \mathrm{P}^{t} \mathrm{Bu}_{2}$ was added to a stirring solution of $\mathrm{ThCl}_{4}(\mathrm{DME})_{2}$ and allowed to stir for $14 \mathrm{~h}$ at room temperature. The solvent removed under vacuum, extracted with toluene, and filtered over Celite. The toluene was removed under vacuum to yield a pale yellow residue which was extracted with THF, filtered over Celite and concentrated to yield a white microcrystalline solid (122 mg, 61\%). X-ray quality crystals were grown from a concentrated THF/hexanes mixture at room temperature. ${ }^{1} \mathrm{H}$ NMR $\left(\mathrm{C}_{6} \mathrm{D}_{6}, 25{ }^{\circ} \mathrm{C}\right)$ : $\delta 1.45\left(\mathrm{~d},{ }^{3} J_{\mathrm{P}-\mathrm{H}}=17.0 \mathrm{~Hz}, 72 \mathrm{H}, \mathrm{C}\left(\mathrm{C} H_{3}\right)_{3}\right) .{ }^{13} \mathrm{C}\left\{{ }^{1} \mathrm{H}\right\}\left(\mathrm{C}_{6} \mathrm{D}_{6}, 25{ }^{\circ} \mathrm{C}\right): \delta 42.21\left(\mathrm{~d},{ }^{1} J_{\mathrm{P}-\mathrm{C}}=18.0 \mathrm{~Hz}\right)$, 28.29. ${ }^{31} \mathrm{P}\left\{{ }^{1} \mathrm{H}\right\}\left(\mathrm{C}_{6} \mathrm{D}_{6}, 25{ }^{\circ} \mathrm{C}\right): \delta 78.3\left(\mathrm{~s}+\mathrm{d}\right.$ satellites, $\left.{ }^{1} J_{\text {Se-P }}=491 \mathrm{~Hz}\right) .{ }^{77} \mathrm{Se}\left\{{ }^{1} \mathrm{H}\right\}\left(\mathrm{C}_{6} \mathrm{D}_{6}, 25{ }^{\circ} \mathrm{C}\right): \delta$ $90.5\left(\mathrm{~d},{ }^{1} J_{\mathrm{P}-\mathrm{Se}}=491 \mathrm{~Hz}\right) . \mathrm{IR}\left(\mathrm{cm}^{-1}\right): 2984$ (s), 2950 (s), 2913 (s), 2860 (s), 1473 (s), 1389 (m), $1364(\mathrm{~s}), 1261$ (w), 1175 (s), 1096 (m), 1071 (m), 1021 (m), 938 (w), 802 (m), 583 (s), $534(\mathrm{~s})$, 483 (s). Anal. calcd. for $\mathrm{C}_{32} \mathrm{H}_{72} \mathrm{P}_{4} \mathrm{Se}_{8} \mathrm{Th} \cdot \mathrm{C}_{4} \mathrm{H}_{8} \mathrm{O}$ : C, 28.51\%; H, 5.32\%. Found C, 28.74\%; H, $5.09 \%$.

$\mathbf{U}\left(\mathbf{S}_{2} \mathbf{P}^{i} \mathbf{P r}_{2}\right)_{4}, \mathbf{1 S}_{-}{ }^{i} \mathbf{P r}$. A $20 \mathrm{~mL}$ scintillation vial was charged with $\mathrm{UCl}_{4}$ (99 mg, $\left.0.261 \mathrm{mmol}\right)$ and THF $(3 \mathrm{~mL})$. A second $20 \mathrm{~mL}$ scintillation vial was charged with $\mathrm{KS}_{2} \mathrm{P}^{i} \operatorname{Pr}_{2}$ (235 $\mathrm{mg}, 1.07$ mmol) and THF (3 mL). Both vials were placed in a freezer at $-20{ }^{\circ} \mathrm{C}$ for $10 \mathrm{~min}$. The $\mathrm{KS}_{2} \mathrm{P}^{i} \operatorname{Pr}_{2}$ 
was added to a stirring solution of $\mathrm{UCl}_{4}$ and underwent a color change to green. The reaction was allowed to warm to room temperature and stirred for $5 \mathrm{~h}$. The solvent was removed under reduced pressure and the solid was extracted with toluene, filtered over Celite, concentrated and layered with diethyl ether. Green X-ray quality crystals were grown at room temperature (158 mg, 63\%). ${ }^{1} \mathrm{H}$ NMR $\left(\mathrm{C}_{6} \mathrm{D}_{6}, 25^{\circ} \mathrm{C}\right): \delta 8.00\left(\mathrm{~s}, \mathrm{br}, 8 \mathrm{H}, \mathrm{CH}\left(\mathrm{CH}_{3}\right)_{2}\right), 4.20\left(\mathrm{~s}, \mathrm{br}, 48 \mathrm{H}, \mathrm{CH}\left(\mathrm{CH}_{3}\right)_{2}\right)$. ${ }^{31} \mathrm{P}\left\{{ }^{1} \mathrm{H}\right\}\left(\mathrm{C}_{6} \mathrm{D}_{6}, 25{ }^{\circ} \mathrm{C}\right): \delta-436.3 . \mathrm{IR}\left(\mathrm{cm}^{-1}\right): 2962(\mathrm{~s}), 2927(\mathrm{~m}), 2869(\mathrm{~m}), 1458(\mathrm{~s}), 1384(\mathrm{~s})$, 1244 (m), $1151(\mathrm{w}), 1088(\mathrm{~m}), 1048(\mathrm{~m}), 1025(\mathrm{~m}), 941(\mathrm{w}), 879(\mathrm{~s}), 838(\mathrm{~m}), 724(\mathrm{w}), 673(\mathrm{~s})$, 646 (s), 505 (m). Anal. calcd. for $\mathrm{C}_{24} \mathrm{H}_{56} \mathrm{P}_{4} \mathrm{~S}_{8} \mathrm{U}$ : C, 29.93\%; H, 5.86\%. Found C, 30.25\%; H, $5.81 \%$.

$\mathbf{U}\left(\mathbf{S}_{2} \mathbf{P}^{t} \mathbf{B u}_{2}\right)_{4}$, 2S-U- ${ }^{t} \mathbf{B u}$. A $20 \mathrm{~mL}$ scintillation vial was charged with $\mathrm{UCl}_{4}(88 \mathrm{mg}, 0.232 \mathrm{mmol})$ and THF $(3 \mathrm{~mL})$. A second $20 \mathrm{~mL}$ scintillation vial was charged with $\mathrm{KS}_{2} \mathrm{P}^{t} \mathrm{Bu}_{2}(237 \mathrm{mg}, 0.954$ $\mathrm{mmol})$ and THF $(4 \mathrm{~mL})$ and placed in a freezer at $-20{ }^{\circ} \mathrm{C}$ for $20 \mathrm{~min}$. The $\mathrm{KS}_{2} \mathrm{P}^{t} \mathrm{Bu}_{2}$ was added to a stirring solution of $\mathrm{UCl}_{4}$ and resulted in a green/yellow color change. The reaction mixture was allowed to warm to room temperature and stirred for $17 \mathrm{~h}$. The solvent was removed under vacuum, extracted with toluene, filtered over a bed of Celite, and solvent removed to yield a yellow precipitate $(134 \mathrm{mg}, 54 \%) .{ }^{1} \mathrm{H}$ NMR $\left(\mathrm{C}_{6} \mathrm{D}_{6}, 25{ }^{\circ} \mathrm{C}\right): \delta-11.90 .\left(\mathrm{s}\right.$, br, $\left.72 \mathrm{H}, \mathrm{C}\left(\mathrm{CH}_{3}\right)_{3}\right)$. ${ }^{31} \mathrm{P}\left\{{ }^{1} \mathrm{H}\right\}\left(\mathrm{C}_{6} \mathrm{D}_{6}, 25{ }^{\circ} \mathrm{C}\right): \delta-467.2 . \mathrm{IR}\left(\mathrm{cm}^{-1}\right): 2988(\mathrm{~m}), 2965(\mathrm{~m}), 2900(\mathrm{~m}), 2873(\mathrm{~m}), 1473(\mathrm{~s})$, 1393 (m), 1360 (m), 1180 (s), 1089 (s), 1082 (s), 939 (m), 804 (m), 621 (s), 518 (m), 466 (m). Anal. calcd. for $\mathrm{C}_{32} \mathrm{H}_{72} \mathrm{P}_{4} \mathrm{~S}_{8} \mathrm{U}$ : C, 35.74\%; H, 6.75\%. Found C, 35.90\%; H, 6.82\%.

$\mathbf{U}\left(\mathbf{S e}_{2} \mathbf{P}^{i} \mathbf{P r}_{2}\right)_{4}$, $\mathbf{1 S e - U -}{ }^{i} \mathbf{P r}$. A $20 \mathrm{~mL}$ scintillation vial was charged with $\mathrm{UI}_{4}(1,4-\text { dioxane })_{2}(138$ $\mathrm{mg}, 0.150) \mathrm{mmol})$. A second $20 \mathrm{~mL}$ scintillation vial was charged with $\mathrm{KSe}_{2} \mathrm{P}^{i} \mathrm{Pr}_{2}(192 \mathrm{mg}$, $0.611 \mathrm{mmol})$ and acetonitrile $(4 \mathrm{~mL})$. The solution of $\mathrm{KSe}_{2} \mathrm{P}^{i} \operatorname{Pr}_{2}$ was placed in a freezer at -20 ${ }^{\circ} \mathrm{C}$ for 20 min. The $\mathrm{UI}_{4}(1,4 \text {-dioxane })_{2}$ was added as a solid to a stirring solution of $\mathrm{KSe}_{2} \mathrm{P}^{i} \mathrm{Pr}_{2}$ and 
resulted in a red color change. The reaction mixture was allowed to stir at room temperature for 5 $\mathrm{h}$, filtered over Celite, and the solvent removed under vacuum to yield a red precipitate. The precipitate was extracted with diethyl ether, filtered over Celite, and the solvent reduced to yield a red precipitate (109 mg, 55\%). X-ray quality crystals were grown from a concentrated diethyl ether solution at room temperature. ${ }^{1} \mathrm{H}$ NMR $\left(\mathrm{C}_{6} \mathrm{D}_{6}, 25{ }^{\circ} \mathrm{C}\right): \delta 7.34\left(\mathrm{~s}, \mathrm{br}, 8 \mathrm{H}, \mathrm{CH}\left(\mathrm{CH}_{3}\right)_{2}\right), 4.37$ (s, br, 48H, CH(CH3 $\left.)_{2}\right) .{ }^{31} \mathrm{P}\left\{{ }^{1} \mathrm{H}\right\}\left(\mathrm{C}_{6} \mathrm{D}_{6}, 25^{\circ} \mathrm{C}\right): \delta-618.0\left(\mathrm{~s}+\mathrm{d}\right.$ satellites, $\left.{ }^{1} J_{\text {Se-P }}=497 \mathrm{~Hz}\right) . \mathrm{IR}$ (cm $\left.{ }^{-1}\right): 2959$ (s), 2919 (s), 2867 (s) 1467 (s), 1382 (m), 1359 (m), 1234 (m), 1156 (m), 1087 (m), 1038 (m), 1018 (m), 920 (w), 875 (m), 645 (m), 613 (s), 538 (s), 469 (w). Anal. calcd. for $\mathrm{C}_{24} \mathrm{H}_{56} \mathrm{P}_{4} \mathrm{Se}_{8} \mathrm{U}: \mathrm{C}, 21.54 \%$; H, 4.22\%. Found C, 21.91\%; H, 4.08\%.

$\mathbf{U}\left(\mathbf{S e}_{2} \mathbf{P}^{t} \mathbf{B u}_{2}\right)_{4}, \mathbf{2 S e}^{-\mathbf{U}^{t}}{ }^{\boldsymbol{B}} \mathbf{B u}$. A $20 \mathrm{~mL}$ scintillation vial was charged with $\mathrm{UCl}_{4}(65 \mathrm{mg}, 0.171$ mmol) and acetonitrile $(2 \mathrm{~mL})$. A second $20 \mathrm{~mL}$ scintillation vial was charged with $\mathrm{KSe}_{2} \mathrm{P}^{t} \mathrm{Bu}_{2}$ (241 mg, $0.704 \mathrm{mmol})$ and acetonitrile $(3 \mathrm{~mL})$. Both vials were placed in $-20{ }^{\circ} \mathrm{C}$ freezer for 20 min. The $\mathrm{KSe}_{2} \mathrm{P}^{t} \mathrm{Bu}_{2}$ was added to the $\mathrm{UCl}_{4}$ and the reaction mixture was allowed to stir for $3 \mathrm{~h}$. The reaction mixture was centrifuged and the orange precipitate was dried, extracted with toluene, and filtered over Celite to yield a red solution. X-ray quality crystals were grown from a concentrated toluene solution at $-20{ }^{\circ} \mathrm{C}(198 \mathrm{mg}, 80 \%) .{ }^{1} \mathrm{H} \mathrm{NMR}\left(\mathrm{C}_{6} \mathrm{D}_{6}, 25{ }^{\circ} \mathrm{C}\right): \delta 2.74\left(\mathrm{~d},{ }^{3} J_{\mathrm{P}-\mathrm{H}}\right.$ $\left.=9.0 \mathrm{~Hz}, 72 \mathrm{H}, \mathrm{C}\left(\mathrm{CH}_{3}\right)_{3}\right) .{ }^{31} \mathrm{P}\left\{{ }^{1} \mathrm{H}\right\}\left(\mathrm{C}_{6} \mathrm{D}_{6}, 25{ }^{\circ} \mathrm{C}\right): \delta-473.0 . \mathrm{IR}\left(\mathrm{cm}^{-1}\right): 2961(\mathrm{~m}), 2936(\mathrm{~m}), 2900$ (m), 2859 (m), $1470(\mathrm{~m}), 1388(\mathrm{~m}), 1362(\mathrm{~m}), 1173(\mathrm{~m}), 1095(\mathrm{~m}), 1070(\mathrm{~m}), 1020(\mathrm{~m}), 929(\mathrm{~m})$, ${ }_{8} 81(\mathrm{~m}), 586(\mathrm{~m}), 566(\mathrm{~s}), 536(\mathrm{~m}), 484(\mathrm{~m})$. Anal. calcd. for $\mathrm{C}_{32} \mathrm{H}_{72} \mathrm{P}_{4} \mathrm{Se}_{8} \mathrm{U} \cdot\left(\mathrm{C}_{7} \mathrm{H}_{8}\right): \mathrm{C}, 30.36 \%$; H, 5.23\%. Found C, 30.63\%; H, 5.23\%.

$\mathbf{U}\left(\mathbf{S e}_{2} \mathbf{P}^{i} \mathbf{P r}_{2}\right)_{3} \mathbf{C l}, 3$ Se-U ${ }^{i P r}$-Cl. A $20 \mathrm{~mL}$ scintillation vial was charged with $\mathrm{UCl}_{4}(152 \mathrm{mg}, 0.400$ mmol) and THF (2 mL). A second $20 \mathrm{~mL}$ scintillation vial was charged with $\mathrm{KSe}_{2} \mathrm{P}^{i} \operatorname{Pr}_{2}(390 \mathrm{mg}$, $1.24 \mathrm{mmol})$ and THF $(3 \mathrm{~mL})$. Both vials were placed in $-23{ }^{\circ} \mathrm{C}$ freezer for $10 \mathrm{~min}$ and $\mathrm{KSe}_{2} \mathrm{P}^{i} \operatorname{Pr}_{2}$ 
was added to $\mathrm{UCl}_{4}$ with an immediate color change to dark red. The reaction was allowed to stir for $5 \mathrm{~h}$. The solvent was removed under vacuum and the red precipitate was extracted with toluene, filtered over a bed of Celite, and concentrated to yield a red microcrystalline solid (352 mg, 80\%). X-ray quality crystals were grown from a concentrated diethyl ether solution at -23 ${ }^{\circ} \mathrm{C} .{ }^{1} \mathrm{H}$ NMR $\left(\mathrm{C}_{6} \mathrm{D}_{6}, 25{ }^{\circ} \mathrm{C}\right): \delta 7.33$ (s, br, 6H, $\left.\left.\mathrm{CH}\left(\mathrm{CH}_{3}\right)_{2}\right), 4.36\left(\mathrm{~s}, \mathrm{br}, 36 \mathrm{H}, \mathrm{CH}\left(\mathrm{CH}_{3}\right)\right)_{2}\right) .{ }^{31} \mathrm{P}\left\{{ }^{1} \mathrm{H}\right\}$ $\left(\mathrm{C}_{6} \mathrm{D}_{6}, 25^{\circ} \mathrm{C}\right): \delta-610.2\left(\mathrm{~s}+\mathrm{d}\right.$ satellites, $\left.{ }^{1} J_{\mathrm{Se}-\mathrm{P}}=487 \mathrm{~Hz}\right) . \mathrm{IR}\left(\mathrm{cm}^{-1}\right): 2959(\mathrm{~s}), 2925(\mathrm{~m}), 2866$ (m), 1459 (s), 1387 (s), 1236 (m), 1158 (w), 1090 (m), 1047 (m), 1023 (m), 925 (w), 878 (m), $837 \quad(w), \quad 649 \quad(\mathrm{~s}), \quad 615 \quad(\mathrm{~s}), \quad 535 \quad(\mathrm{~s}), \quad 473 \quad$ (m). Anal. $\quad \begin{array}{llllll}\text { calcd. } & \text { for }\end{array}$ $\mathrm{C}_{18} \mathrm{H}_{42} \mathrm{ClP}_{3} \mathrm{Se}_{6} \mathrm{U} \cdot 0.5\left(\mathrm{Et}_{2} \mathrm{O}\right) \bullet 0.5\left(\mathrm{C}_{7} \mathrm{H}_{8}\right): \mathrm{C}, 21.07 \% ; \mathrm{H}, 3.84 \%$. Found C, 21.38\%; H, 3.85\%.

$\mathbf{U}\left(\mathbf{S}_{2} \mathbf{P}^{t} \mathbf{B u}\right)_{3} \mathbf{C l}$, 4S-U ${ }^{\text {tBu}}$-Cl. Method $A$. A $20 \mathrm{~mL}$ scintillation vial was charged with $\mathrm{UCl}_{4}(76$ $\mathrm{mg}, 0.200 \mathrm{mmol})$ and $\mathrm{CH}_{3} \mathrm{CN}(3 \mathrm{~mL})$. A second $20 \mathrm{~mL}$ scintillation vial was charged with $\mathrm{KS}_{2} \mathrm{P}^{\mathrm{t}} \mathrm{Bu}_{2}(154 \mathrm{mg}, 0.620 \mathrm{mmol})$ and $\mathrm{CH}_{3} \mathrm{CN}(3 \mathrm{~mL})$. Both vials were placed in a $-23{ }^{\circ} \mathrm{C}$ freezer for $30 \mathrm{~min}$ and the $\mathrm{KS}_{2} \mathrm{P}^{\mathrm{t}} \mathrm{Bu}_{2}$ was added to the $\mathrm{UCl}_{4}$ mixture and allowed to stir for $14 \mathrm{~h}$ at room temperature to yield a blue/green mixture. The solvent was removed under vacuum and extracted with toluene $(2 \times 5 \mathrm{~mL})$, filtered over Celite, concentrated, and placed in a $-23{ }^{\circ} \mathrm{C}$ freezer to yield a green precipitate $(124 \mathrm{mg}, 69 \%)$. X-ray quality crystals were grown from a concentrated toluene/diethyl ether solution at $-23{ }^{\circ} \mathrm{C} .{ }^{1} \mathrm{H}$ NMR $\left(\mathrm{C}_{6} \mathrm{D}_{6}, 25{ }^{\circ} \mathrm{C}\right): \delta-3.36\left(\mathrm{~s}, \mathrm{br}, 54 \mathrm{H}, \mathrm{C}\left(\mathrm{CH}_{3}\right)_{3}\right)$. ${ }^{31} \mathrm{P}\left\{{ }^{1} \mathrm{H}\right\}\left(\mathrm{C}_{6} \mathrm{D}_{6}, 25^{\circ} \mathrm{C}\right): \delta-495.0$.

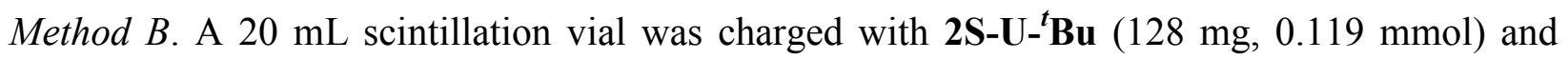
THF (5 mL). A second $20 \mathrm{~mL}$ scintillation vial was charged with $\mathrm{ZnCl}_{2}$ (8 mg, $\left.0.0595 \mathrm{mmol}\right)$

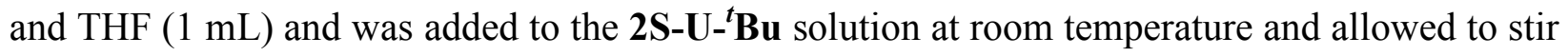
for $14 \mathrm{~h}$ to yield a green/yellow solution. The solvent was removed under vacuum, extracted with 
toluene, filtered over Celite, concentrated a placed in a $-23{ }^{\circ} \mathrm{C}$ freezer to yield a green precipitate (59 mg, 55\%). NMR spectroscopy data matched spectra reported for Method A.

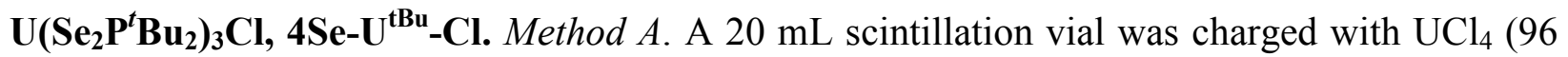
$\mathrm{mg}, 0.254 \mathrm{mmol})$ and $\mathrm{CH}_{3} \mathrm{CN}(3 \mathrm{~mL})$. A second $20 \mathrm{~mL}$ scintillation vial was charged with $\mathrm{KSe}_{2} \mathrm{P}^{t} \mathrm{Bu}_{2}(269 \mathrm{mg}, 0.786 \mathrm{mmol})$ and was added as solid to $\mathrm{UCl}_{4}$ at room temperature. The reaction was allowed to stir for $14 \mathrm{~h}$ to yield an orange/red precipitate. The solvent was decanted and the solid was dried under vacuum. The orange/red solid was extracted with toluene $(2 \times 4$ $\mathrm{mL}$ ), filtered over Celite, concentrated, and placed in a $-23{ }^{\circ} \mathrm{C}$ freezer to yield a red precipitate (234 mg, 78\%). X-ray quality crystals were grown from a concentrated toluene solution at -23 ${ }^{\circ} \mathrm{C} .{ }^{1} \mathrm{H}$ NMR $\left(\mathrm{C}_{6} \mathrm{D}_{6}, 25{ }^{\circ} \mathrm{C}\right): \delta-2.31\left(\mathrm{~s}, \mathrm{br}, 54 \mathrm{H}, \mathrm{C}\left(\mathrm{CH}_{3}\right)_{3}\right) .{ }^{31} \mathrm{P}\left\{{ }^{1} \mathrm{H}\right\}\left(\mathrm{C}_{6} \mathrm{D}_{6}, 25{ }^{\circ} \mathrm{C}\right): \delta-690.0$. IR $\left(\mathrm{cm}^{-1}\right): 2981$ (s), 2960 (s) 2941 (s), 2911 (s), 2899 (s), 2866 (m), 1470 (s), 1389 (m), 1363 (s), 1171 (s), 1020 (s), 936 (m), 799 (m), 606 (w), 583 (s), 532 (s), 487 (s). Anal. calcd. for $\mathrm{C}_{24} \mathrm{H}_{54} \mathrm{ClP}_{3} \mathrm{Se}_{6} \mathrm{U}$ : C, 24.37\%; H, 4.60\%. Found C, 24.77\%; H, 4.31\%.

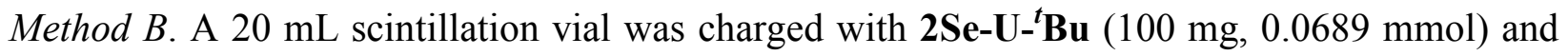
THF (3 mL). A second $20 \mathrm{~mL}$ scintillation vial was charged with $\mathrm{ZnCl}_{2}(4.7 \mathrm{mg}, 0.0345 \mathrm{mmol})$ and added as solid to $\mathbf{2 S e}-\mathbf{U}-{ }^{t} \mathbf{B u}$ at room temperature. The reaction was stirred for $14 \mathrm{~h}$ to yield a dark red solution. The reaction was filtered over Celite and the solvent was removed under vacuum. The dark red solid was washed with hexanes $(2 \times 3 \mathrm{~mL})$ and dried. The red solid was extracted with toluene, filtered over Celite, concentrated and placed in a $-23{ }^{\circ} \mathrm{C}$ freezer to yield a red precipitate (43 mg, 53\%). NMR spectroscopy data matched spectra reported for Method A.

$\mathbf{U}\left(\mathbf{S e}_{2} \mathbf{P}^{t} \mathbf{B u}_{2}\right)_{3} \mathbf{F}, \mathbf{5 S e}^{\mathrm{tBu}}-\mathbf{F}$. A $20 \mathrm{~mL}$ scintillation vial was charged with $\mathbf{2 S e}-\mathbf{U}^{{ }^{t}} \mathbf{B u}(174 \mathrm{mg}$, $0.120 \mathrm{mmol})$ and THF $(3 \mathrm{~mL})$. A second $20 \mathrm{~mL}$ scintillation vial was charged with $\mathrm{Hg}_{2} \mathrm{~F}_{2}(26$ 


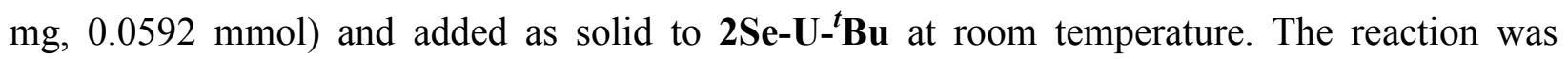
allowed to stir for $14 \mathrm{~h}$ to yield an orange solution. The reaction was filtered over Celite, concentrated, layered with hexane, and placed in a $-23{ }^{\circ} \mathrm{C}$ freeze overnight to yield an orange precipitate $(85 \mathrm{mg}, 61 \%) .{ }^{1} \mathrm{H} \mathrm{NMR}\left(\mathrm{C}_{6} \mathrm{D}_{6}, 25{ }^{\circ} \mathrm{C}\right): \delta-2.71\left(\mathrm{~s}, \mathrm{br}, 54 \mathrm{H}, \mathrm{C}(\mathrm{CH})_{3}\right) .{ }^{31} \mathrm{P}\left\{{ }^{1} \mathrm{H}\right\}\left(\mathrm{C}_{6} \mathrm{D}_{6}\right.$, $\left.25^{\circ} \mathrm{C}\right): \delta-786.0 . \mathrm{IR}\left(\mathrm{cm}^{-1}\right): 2953$ (s), 2907 (s), 2863 (s), 1463 (s), 1393 (w), 1361 (s), $1171(\mathrm{~s})$, $1099(\mathrm{~m}), 1071(\mathrm{~m}), 1020(\mathrm{~s}), 937(\mathrm{w}), 816(\mathrm{w}), 801(\mathrm{~m}), 575(\mathrm{~s}), 524(\mathrm{~s}), 483$ (s). Due to the similar solubility of $\mathbf{5 S e}-\mathbf{U}^{\mathbf{t B u}}-\mathbf{F}$ and the transmetalation byproduct, a suitable element analysis could not be achieved.

$\mathbf{U}\left(\mathbf{S e}_{2} \mathbf{P}^{t} \mathbf{B u}_{2}\right)_{3} \mathbf{B r}, \mathbf{6 S e}^{\mathrm{tBu}}-\mathbf{B r}$. A $20 \mathrm{~mL}$ scintillation vial was charged with $\mathbf{2 S e - U}-{ }^{\boldsymbol{t}} \mathbf{B u}(175 \mathrm{mg}$, $0.121 \mathrm{mmol})$ and THF $(5 \mathrm{~mL})$. A second $20 \mathrm{~mL}$ scintillation vial was charged with $\mathrm{CuBr}(17 \mathrm{mg}$,

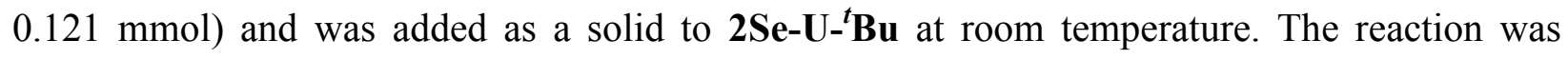
stirred for $14 \mathrm{~h}$, filtered over Celite, concentrated, layered with hexane, and placed in a $-23{ }^{\circ} \mathrm{C}$ freeze overnight to yield a dark red precipitate $(92 \mathrm{mg}, 62 \%) .{ }^{1} \mathrm{H}$ NMR $\left(\mathrm{C}_{6} \mathrm{D}_{6}, 25{ }^{\circ} \mathrm{C}\right): \delta-1.39(\mathrm{~s}$, br, 54H, $\left.\mathrm{C}\left(\mathrm{CH}_{3}\right)_{3}\right) .{ }^{31} \mathrm{P}\left\{{ }^{1} \mathrm{H}\right\}\left(\mathrm{C}_{6} \mathrm{D}_{6}, 25^{\circ} \mathrm{C}\right): \delta-652.0$. IR $\left(\mathrm{cm}^{-1}\right): 2954$ (s), 2098 (s), 2864 (s), 1464 (s), $1390(\mathrm{w}), 1362(\mathrm{~m}), 1172(\mathrm{~s}), 1070(\mathrm{~m}), 1021(\mathrm{~s}), 936(\mathrm{w}), 841(\mathrm{w}), 802(\mathrm{~m}), 581(\mathrm{~m}), 530$ (m), 484 (s). Due to the similar solubility of $\mathbf{6 S e}-\mathbf{U}^{\mathrm{tBu}}-\mathbf{B r}$ and the transmetalation byproduct, a suitable element analysis could not be achieved.

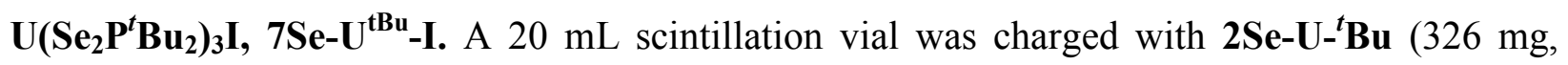
$0.225 \mathrm{mmol})$ and THF (5 mL). A second $20 \mathrm{~mL}$ scintillation vial was charged with $\mathrm{CuI}(86 \mathrm{mg}$, $0.856 \mathrm{mmol}$ ) and added as a solid to $\mathbf{2} \mathbf{S e}-\mathbf{U}^{-}{ }^{t} \mathbf{B u}$ at room temperature. The reaction was stirred for $14 \mathrm{~h}$ and filtered over Celite, and the solvent was removed under vacuum. The red solid was extracted with hexanes $(2 \times 3 \mathrm{~mL})$, decanted, and the red precipitate was dried under vacuum. The red solid was extracted with toluene, filtered over Celite, concentrated, and placed in a -23 
${ }^{\circ} \mathrm{C}$ freeze overnight to yield a dark red precipitate (195 mg, 68\%). X-ray quality crystals were grown from a toluene/hexanes mixture at $-23{ }^{\circ} \mathrm{C} .{ }^{1} \mathrm{H}$ NMR $\left(\mathrm{C}_{6} \mathrm{D}_{6}, 25{ }^{\circ} \mathrm{C}\right): \delta-0.30(\mathrm{~s}, \mathrm{br}, 54 \mathrm{H}$, $\left.\mathrm{C}\left(\mathrm{CH}_{3}\right)_{3}\right) .{ }^{31} \mathrm{P}\left\{{ }^{1} \mathrm{H}\right\}\left(\mathrm{C}_{6} \mathrm{D}_{6}, 25{ }^{\circ} \mathrm{C}\right): \delta$-635.0. IR $\left(\mathrm{cm}^{-1}\right): 2960$ (s), 2914 (s), 2899 (s), 2863 (s), 1471 (s), 1389 (m), 1364 (s), 1172 (s), 1079 (m), 1020 (s), 936 (m), 800 (s), 636 (w), 606 (w), 583 (s), 531 (s), 483 (s). Anal. calcd. for $\mathrm{C}_{24} \mathrm{H}_{54} \mathrm{IP}_{3} \mathrm{Se}_{6} \mathrm{U}$ : C, 22.62\%; H, 4.27\%. Found C, $22.60 \% ; \mathrm{H}, 4.02 \%$.

$\left[\mathbf{C u}\left(\mathbf{S e}_{2} \mathbf{P}^{t} \mathbf{B u}_{2}\right)\right]_{4}, \mathbf{8 S e}-\mathbf{C u}-{ }^{t} \mathbf{B u}$. A $20 \mathrm{~mL}$ scintillation vial was charged with $\left[\mathrm{Cu}(\mathrm{NCMe})_{4}\right]\left[\mathrm{PF}_{6}\right]$ (109 mg, $0.292 \mathrm{mmol})$ and acetonitrile $(3 \mathrm{~mL}) . \mathrm{KSe}_{2} \mathrm{P}^{t} \mathrm{Bu}_{2}(100 \mathrm{mg}, 0.292 \mathrm{mmol})$ was added to the mixture as a solid at room temperature and the reaction was allowed to stir for $14 \mathrm{~h}$. The solvent was removed under vacuum and the solid was extracted with toluene, filtered over Celite and concentrated. X-ray quality yellow crystals were grown from toluene/hexanes mixture at -23 ${ }^{\circ} \mathrm{C}$ (two crops, $\left.80 \mathrm{mg}, 75 \%\right) .{ }^{1} \mathrm{H}$ NMR $\left(\mathrm{C}_{6} \mathrm{D}_{6}, 25{ }^{\circ} \mathrm{C}\right): \delta 1.51\left(\mathrm{~d},{ }^{3} J_{\mathrm{P}-\mathrm{H}}=17.0 \mathrm{~Hz}, 72 \mathrm{H}, \mathrm{C}\left(\mathrm{CH}_{3}\right)_{3}\right)$. ${ }^{13} \mathrm{C}\left\{{ }^{1} \mathrm{H}\right\}\left(\mathrm{C}_{6} \mathrm{D}_{6}, 25{ }^{\circ} \mathrm{C}\right): \delta 42.18\left(\mathrm{~d},{ }^{1} J_{\mathrm{P}-\mathrm{C}}=18.0 \mathrm{~Hz}\right), 29.32 .{ }^{31} \mathrm{P}\left\{{ }^{1} \mathrm{H}\right\}\left(\mathrm{C}_{6} \mathrm{D}_{6}, 25{ }^{\circ} \mathrm{C}\right): \delta 90.6(\mathrm{~s}+\mathrm{d}$, satellites, $\left.{ }^{1} J_{\mathrm{Se}-\mathrm{P}}=533 \mathrm{~Hz}\right)$. IR (cm $\left.{ }^{-1}\right): 2995$ (s), 2978 (s), 2961 (s), 2913 (s), 2867 (s), 1465 (s), $1388(\mathrm{~m}), 1362$ (s), 1173 (s), 1020 (s), 937 (w), 801 (s), $730(\mathrm{~m}), 695(\mathrm{w}), 596(\mathrm{w}), 577(\mathrm{~s}), 528$ (s), 484 (s). Anal. calcd. for $\mathrm{C}_{32} \mathrm{H}_{72} \mathrm{P}_{4} \mathrm{Se}_{8} \mathrm{Cu}_{4} \bullet 0.5\left(\mathrm{C}_{7} \mathrm{H}_{8}\right)$ : C, 28.19\%; H, 5.06\%. Found C, $28.47 \% ; \mathrm{H}, 5.04 \%$.

Table 1. X-ray crystallographic data shown for complexes homoleptic $\mathrm{Th}\left(\mathrm{E}_{2} \mathrm{PR}_{2}\right)_{4}, \mathrm{E}=\mathrm{S}, \mathrm{Se} ; \mathrm{R}$ $={ }^{i} \mathrm{Pr},{ }^{t} \mathrm{Bu}$; and $\mathrm{U}\left(\mathrm{S}_{2} \mathrm{P}^{i} \mathrm{Pr}\right)_{4}$

\begin{tabular}{|c|c|c|c|c|c|}
\hline & 1S-Th- ${ }^{i} \mathrm{Pr}$ & $2 S-T h-{ }^{t} B u$ & 1Se-Th- ${ }^{i} \mathrm{Pr}$ & 2Se-Th- ${ }^{t} \mathrm{Bu}$ & $1 S-U-{ }^{i} \mathbf{P r}$ \\
\hline Empirical formula & $\mathrm{C}_{28} \mathrm{H}_{64} \mathrm{OP}_{4} \mathrm{~S}_{8} \mathrm{Th}$ & $\mathrm{C}_{32} \mathrm{H}_{72} \mathrm{P}_{4} \mathrm{~S}_{8} \mathrm{Th}$ & $\mathrm{C}_{24} \mathrm{H}_{56} \mathrm{P}_{4} \mathrm{Se}_{8} \mathrm{Th}$ & $\mathrm{C}_{32} \mathrm{H}_{72} \mathrm{P}_{4} \mathrm{Se}_{8} \mathrm{Th}$ & $\mathrm{C}_{24} \mathrm{H}_{56} \mathrm{P}_{4} \mathrm{~S}_{8} \mathrm{U}$ \\
\hline Crystal habit, color & Brick, colorless & Prism, colorless & Brick, colorless & Prism, colorless & Needle, green \\
\hline Temperature $(\mathrm{K})$ & $100(2)$ & $100(2)$ & $173(2)$ & $100(2)$ & $100(2)$ \\
\hline Space group & $P 2_{1} / \mathrm{n}$ & $P-4 n 2$ & $C \mathrm{c}$ & $P-4 \mathrm{n} 2$ & $P$ nn2 \\
\hline
\end{tabular}


20

21

22

\begin{tabular}{|c|c|c|c|c|c|}
\hline$a(\AA)$ & $16.756(2)$ & $18.090(15)$ & $16.854(3)$ & $18.3858(14)$ & $21.242(4)$ \\
\hline$b(\AA)$ & $13.249(1)$ & $18.090(15)$ & $13.348(3)$ & $18.3858(14)$ & $36.150(6)$ \\
\hline$c(\AA)$ & $20.758(2)$ & $15.871(13)$ & $18.538(4)$ & $16.2449(12)$ & $10.995(2)$ \\
\hline$\alpha\left(^{\circ}\right)$ & 90.00 & 90 & 90.00 & 90.00 & 90.00 \\
\hline$\beta\left(^{\circ}\right)$ & $108.461(1)$ & 90 & $96.522(2)$ & 90.00 & 90.00 \\
\hline$\gamma\left(^{\circ}\right)$ & 90.00 & 90 & 90.00 & 90.00 & 90.00 \\
\hline$Z$ & 4 & 4 & 4 & 4 & 4 \\
\hline Calculated density $\left(\mathrm{Mg}^{3} \mathrm{~m}^{3}\right)$ & 1.564 & 1.368 & 2.136 & 1.747 & 1.518 \\
\hline Absorption coefficient $\left(\mathrm{mm}^{-1}\right)$ & 3.962 & 3.336 & 10.796 & 8.153 & 4.407 \\
\hline Final R indices $[\mathrm{I}>2 \sigma(\mathrm{I})]$ & $\mathrm{R}=0.0171$ & $\mathrm{R}=0.0139$ & $\mathrm{R}=0.0209$ & $\mathrm{R}=0.0131$ & $\mathrm{R}=0.0474$ \\
& $\mathrm{R}_{\mathrm{W}}=0.0401$ & $\mathrm{R}_{\mathrm{W}}=0.0315$ & $\mathrm{R}_{\mathrm{W}}=0.0434$ & $\mathrm{R}_{\mathrm{W}}=0.0297$ & $\mathrm{R}_{\mathrm{W}}=0.1272$ \\
\hline
\end{tabular}

Table 2. X-ray crystallographic data shown for complexes $\mathrm{U}\left(\mathrm{Se}_{2} \mathrm{PR}_{2}\right)_{4}, \mathrm{R}={ }^{i} \mathrm{Pr},{ }^{t} \mathrm{Bu}$; $\mathrm{U}\left(\mathrm{Se}_{2} \mathrm{PR}_{2}\right)_{3} \mathrm{Cl}, \mathrm{R}={ }^{i} \mathrm{Pr},{ }^{t} \mathrm{Bu} ; \mathrm{U}\left(\mathrm{Se}_{2} \mathrm{P}^{t} \mathrm{Bu}_{2}\right)_{3} \mathrm{I}$, and $\left[\mathrm{Cu}\left(\mathrm{Se}_{2} \mathrm{P}^{t} \mathrm{Bu}_{2}\right)\right]_{4}$.

\begin{tabular}{|c|c|c|c|c|c|c|}
\hline & $2 \mathrm{Se}-\mathrm{U}-{ }^{i} \mathrm{Pr}$ & $2 \mathrm{Se}-\mathrm{U}^{-}{ }^{t} \mathrm{Bu}$ & $3 \mathrm{Se}-\mathrm{U}^{i \mathrm{Pr}}-\mathrm{Cl}$ & $4 \mathrm{Se}-\mathrm{U}^{\mathrm{tBu}}-\mathrm{Cl}$ & $7 \mathrm{Se}-\mathrm{U}^{\mathrm{tBu}}-\mathrm{I}$ & $8 \mathrm{Se}-\mathrm{Cu}-{ }^{t} \mathrm{Bu}$ \\
\hline CCDC deposit number & 1404310 & 1404312 & 1404309 & 1404311 & 1406600 & 1404303 \\
\hline Empirical formula & $\mathrm{C}_{24} \mathrm{H}_{56} \mathrm{P}_{4} \mathrm{Se}_{8} \mathrm{U}$ & $\mathrm{C}_{32} \mathrm{H}_{72} \mathrm{P}_{4} \mathrm{Se}_{8} \mathrm{U}$ & $\mathrm{C}_{25} \mathrm{H}_{50} \mathrm{ClP}_{3} \mathrm{Se}_{6} \mathrm{U}$ & $\mathrm{C}_{24} \mathrm{H}_{54} \mathrm{ClP}_{3} \mathrm{Se}_{6} \mathrm{U}$ & $\mathrm{C}_{24} \mathrm{H}_{54} \mathrm{IP}_{3} \mathrm{Se}_{6} \mathrm{U}$ & $\mathrm{C}_{32} \mathrm{H}_{72} \mathrm{P}_{4} \mathrm{Se}_{8} \mathrm{Cu}_{4}$ \\
\hline Formula weight $(\mathrm{g} / \mathrm{mol})$ & 1338.27 & 1450.48 & 1190.80 & 1182.82 & 1182.82 & 1466.61 \\
\hline Crystal habit, color & Prism, red & Prism, red & Prism, red & Prism, red & Prism, red & yellow \\
\hline Temperature $(\mathrm{K})$ & $100(2)$ & $100(2)$ & $173(2)$ & $100(2)$ & $100(2)$ & $100(2)$ \\
\hline Space group & $C \mathrm{c}$ & $P-4 \mathrm{n} 2$ & $P 212121$ & $P 212121$ & $P 212121$ & $C 2 / \mathrm{c}$ \\
\hline Volume $\left(\AA^{3}\right)$ & $4069.7(4)$ & $5480.4(9)$ & $3825.3(6)$ & $3843.9(9)$ & $3843.9(9)$ & $5984.5(14)$ \\
\hline$a(\AA)$ & $16.7142(10)$ & $18.3367(14)$ & $12.4588(12)$ & $10.7542(15)$ & $10.7542(15)$ & $20.874(3)$ \\
\hline$b(\AA)$ & $13.2787(8)$ & $18.3367(14)$ & $14.4390(14)$ & $16.203(2)$ & $16.203(2)$ & $18.581(3)$ \\
\hline$c(\AA)$ & $18.4521(11)$ & $16.2992(12)$ & $21.264(2)$ & $22.059(3)$ & $22.059(3)$ & $16.759(2)$ \\
\hline$\alpha\left(^{\circ}\right)$ & 90 & 90 & 90 & 90 & 90 & 90 \\
\hline$\beta\left(^{\circ}\right)$ & $96.4050(10)$ & 90 & 90 & 90 & 90 & $112.976(2)$ \\
\hline$\gamma\left({ }^{\circ}\right)$ & 90 & 90 & 90 & 90 & 90 & 90 \\
\hline $\begin{array}{l}\text { Calculated density } \\
\left(\mathrm{Mg} / \mathrm{m}^{3}\right)\end{array}$ & 2.184 & 1.758 & 2.068 & 2.044 & 2.044 & 1.628 \\
\hline $\begin{array}{l}\text { Absorption coefficient } \\
\left(\mathrm{mm}^{-1}\right)\end{array}$ & 11.316 & 8.411 & 10.162 & 10.112 & 10.112 & 6.393 \\
\hline inal $R$ indices $[I>2 \sigma(I)]$ & $\begin{array}{c}\mathrm{R}=0.0159 \\
\mathrm{R}_{\mathrm{W}}=0.0341\end{array}$ & $\begin{array}{l}\mathrm{R}=0.0145 \\
\mathrm{R}_{\mathrm{W}}=0.0315\end{array}$ & $\begin{array}{c}\mathrm{R}=0.0187 \\
\mathrm{R}_{\mathrm{W}}=0.0386\end{array}$ & $\begin{array}{c}\mathrm{R}=0.0175 \\
\mathrm{R}_{W}=0.0391\end{array}$ & $\begin{array}{c}\mathrm{R}=0.0277 \\
\mathrm{R}_{\mathrm{W}}=0.0647\end{array}$ & $\begin{array}{l}\mathrm{R}=0.0203 \\
\mathrm{R}_{\mathrm{W}}=0.0452\end{array}$ \\
\hline
\end{tabular}

Computational Details. All calculations were performed at the density functional theoretical (DFT) level using version 6.6 of the TURBOMOLE quantum chemistry software package. ${ }^{18}$ XRD-derived structural parameters were used as the basis for geometry optimizations. The hybrid-GGA $\mathrm{PBE}^{19}$ exchange-correlation functional, which incorporates a perturbatively derived $25 \%$ contribution of exact exchange, was used throughout. In all calculations, basis sets of polarized triple- $\zeta$ quality were used. For geometry optimizations, Ahlrichs-style basis sets ${ }^{20,21}$ were employed, incorporating an effective core potential replacing 60 core electrons of the 
actinide ion $^{22}$. We have successfully applied this model chemistry in previous studies of felement complexes. ${ }^{23,24}$ All complexes considered in this study were identified as energetic minima through vibrational frequency analysis.

For subsequent analysis of the electron density, all-electron single point energy calculations were performed at the optimized geometries. These calculations replaced the Ahlrichs basis set on the actinide with the SARC basis set of Pantazis and Neese ${ }^{25}$ and incorporated scalar relativistic effects via the $2^{\text {nd }}$ order Douglas-Kroll-Hess Hamiltonian. Topological and integrated properties of the resulting electron densities were obtained via application of the Quantum Theory of Atoms in Molecules ${ }^{26}$ (QTAIM) as implemented in version 14.11.23 of the AIMAll code. $^{27}$

\section{Results and Discussion}

The potassium salts of the dithio- and diselenophosphinate, $\mathrm{KS}_{2} \mathrm{P}^{t} \mathrm{Bu}_{2}, \mathrm{KSe}_{2} \mathrm{P}^{i} \mathrm{Pr}_{2}$, and $\mathrm{KSe}_{2} \mathrm{P}^{t} \mathrm{Bu}_{2}$ were synthesized using previously reported procedures, i.e. the deprotonation of the secondary phosphine with potassium hydroxide followed by addition of two equivalents of the elemental chalcogen. ${ }^{28}$ All were obtained as white solids in good to excellent yields.

The synthesis of complexes (1S-Th- $\left.{ }^{i} \mathbf{P r}\right)-\left(\mathbf{1 S e}-\mathbf{T h}-{ }^{t} \mathbf{B u}\right)$ was achieved through the stoichiometric salt metathesis reactions at room temperature (eq 1). Compounds (2S-Th- $\left.{ }^{\mathbf{t}} \mathbf{B u}\right)$ (2Se-Th- $\left.{ }^{t} \mathbf{B u}\right)$ have not been reported previously. The salt metathesis reactions of complexes 1STh- ${ }^{i} \mathbf{P r}$ and $2 \mathrm{~S}-\mathbf{T h}-{ }^{t} \mathbf{B u}$ yielded colorless solutions while complexes $\mathbf{1 S e - T h}{ }^{i} \mathbf{P r}$ and $\mathbf{2 S e - T h}-{ }^{t} \mathbf{B u}$ were a yellow hue. The solubility of the diisopropyldichalogenophosphinates and di-tertbutyldichalogenophosphinates was greater in toluene and THF, but not soluble in aliphatic hydrocarbons, diethyl ether, or acetonitrile. Synthesis of the $\mathrm{U}\left[\mathrm{S}_{2} \mathrm{P}^{i} \operatorname{Pr}_{2}\right]_{4}$ (1S-U-U $\left.{ }^{i} \mathbf{P r}\right)$ and 
$\mathrm{U}\left[\mathrm{S}_{2} \mathrm{P}^{t} \mathrm{Bu}_{2}\right]_{4}\left(\mathbf{2} \mathbf{S}-\mathbf{U}-{ }^{t} \mathbf{B u}\right)$ was accomplished through the salt metathesis reactions of $\mathrm{K}\left[\mathrm{S}_{2} \mathrm{PR}_{2}\right], \mathrm{R}$ $={ }^{i} \mathrm{Pr}$ and ${ }^{t} \mathrm{Bu}$, respectively, and $\mathrm{UCl}_{4}$ in THF at $-20{ }^{\circ} \mathrm{C}$ (eq 2 ).

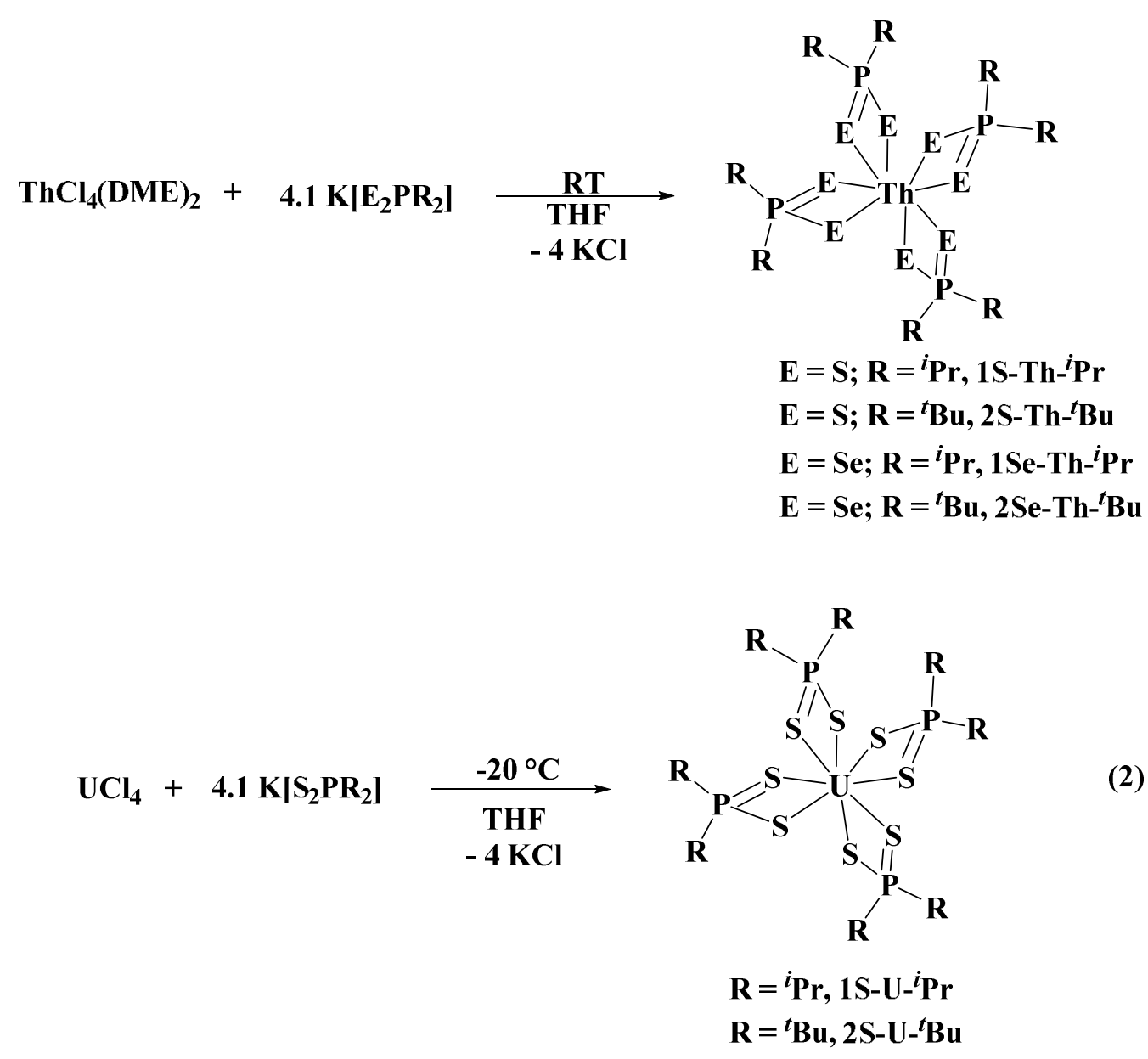

Complex 1S-U- ${ }^{i}$ Pr was recrystallized from concentrated diethyl ether solution to yield green

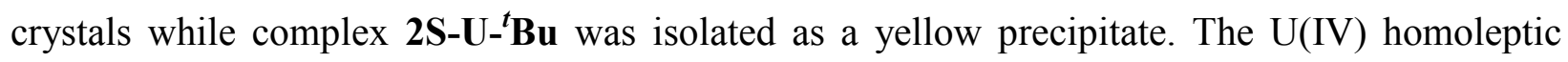
complex was the exclusive product isolated in reasonable yields: $63 \%$ and $54 \%$ for $\mathbf{1 S}-\mathbf{U}_{-}{ }^{i} \mathbf{P r}$ and 2S-U- ${ }^{t} \mathbf{B u}$ respectively. The diisopropyldiselenophosphinate and di-tertbutyldiselenophosphinate ligands demonstrated various reactivity dependent on uranium starting material and solvent used. For example, the reaction between $\mathrm{UCl}_{4}$ and three equivalents of $\mathrm{K}\left[\mathrm{Se}_{2} \mathrm{P}^{i} \mathrm{Pr}_{2}\right]$ in THF at $-20{ }^{\circ} \mathrm{C}$ yielded $\mathrm{U}\left[\mathrm{Se}_{2} \mathrm{P}^{i} \mathrm{Pr}_{2}\right]_{3} \mathrm{Cl}$, 3Se-U ${ }^{i \mathbf{P r}}-\mathbf{C l}$, (eq 3). However, when 
$\mathrm{UI}_{4}(1,4 \text {-dioxane })_{2}$ was used, the homoleptic product, $\mathrm{U}\left[\mathrm{Se}_{2} \mathrm{P}^{i} \operatorname{Pr}_{2}\right]_{4}$, $\mathbf{1 S e - U}-{ }^{i} \mathbf{P r}$, (eq 4) was isolated. Analogous to these reactions, the ${ }^{t} \mathrm{Bu}$ analogs displayed similar coordination, i.e. $\mathrm{U}\left[\mathrm{Se}_{2} \mathrm{P}^{t} \mathrm{Bu}_{2}\right]_{3} \mathrm{Cl}, \mathbf{4} \mathbf{S e}-\mathbf{U}^{t \mathbf{B u}}-\mathbf{C l}\left(\right.$ eq 5 ), and $\mathrm{U}\left[\mathrm{Se}_{2} \mathrm{P}^{t} \mathrm{Bu}_{2}\right]_{4}, \mathbf{2 S e - U}-{ }^{t} \mathbf{B u}$ (eq 4), were obtained with $\mathrm{UCl}_{4}$ and $\mathrm{UI}_{4}(1,4 \text {-dioxane })_{2}$, respectively. The homoleptic complexes can be produced from $\mathrm{UCl}_{4}$ and four equivalents of the respective potassium salts when acetonitrile is used as a solvent. When the reaction between $\mathrm{UCl}_{4}$ and $\mathrm{K}\left[\mathrm{Se}_{2} \mathrm{P}^{t} \mathrm{Bu}_{2}\right]$ was attempted in THF, a bridging $\mu_{2}$-oxo complex, $\mu_{2}-\mathrm{O}\left\{\mathrm{U}\left[\mathrm{Se}_{2} \mathrm{P}^{t} \mathrm{Bu}_{2}\right]_{3}\right\}_{2}$, was isolated (see Supporting Information).

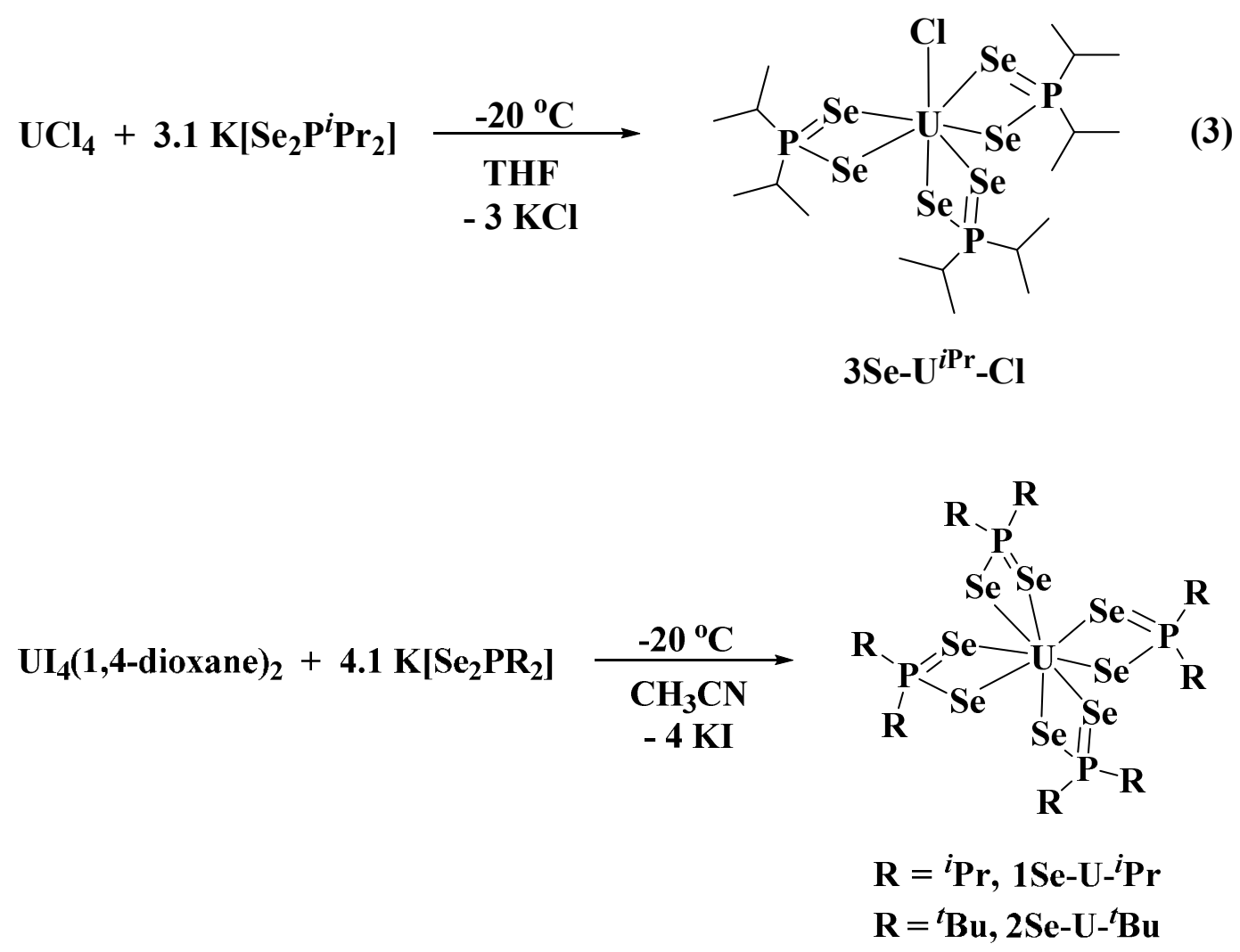




$$
\mathrm{UCl}_{4}+3.1 \mathrm{~K}\left[\mathrm{Se}_{2} \mathrm{P}^{t} \mathrm{Bu}_{2}\right]
$$

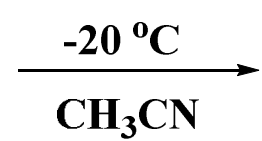

- $3 \mathrm{KCl}$

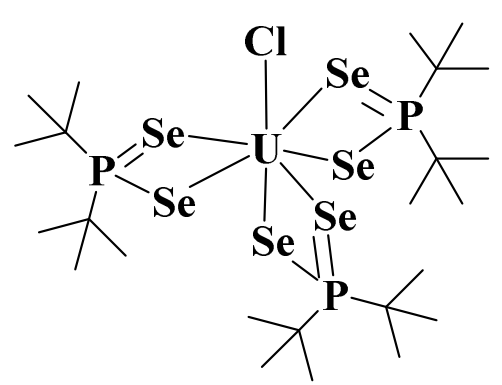

$4 \mathrm{Se}-\mathrm{U}^{t \mathrm{Bu}}-\mathrm{Cl}$

The ${ }^{1} \mathrm{H}$ and ${ }^{13} \mathrm{C}\left\{{ }^{1} \mathrm{H}\right\}$ spectra for complexes (1S-Th- $\left.{ }^{i} \mathbf{P r}\right)-\left(\mathbf{2 S e}-\mathbf{T h}-{ }^{\boldsymbol{t}} \mathbf{B u}\right)$ were similar; however, the ${ }^{31} \mathrm{P}\{\mathrm{H}\}$ spectra displayed two significant shifts (Table 3). The ${ }^{31} \mathrm{P}\left\{{ }^{1} \mathrm{H}\right\}$ chemical resonances of $\mathbf{1 S - T h}-{ }^{i} \mathbf{P r}$ and $\mathbf{2 S - T h}{ }^{t} \mathbf{B u}$ occurred at 84.9 and 99.1 ppm, respectively, while ${ }^{31} \mathrm{P}\left\{{ }^{1} \mathrm{H}\right\}$ chemical resonances of $\mathbf{1 S e}-\mathbf{T h}-{ }^{i} \mathbf{P r}$ and $\mathbf{2 S e}-\mathbf{T h}-{ }^{t} \mathbf{B u}$ occurred at 57.6 and $78.3 \mathrm{ppm}$, respectively. The upfield shift in chemical resonances of $\mathbf{1 S e - T h}-{ }^{i} \mathbf{P r}$ and $2 \mathrm{Se}-\mathbf{T h}-{ }^{\boldsymbol{t}} \mathbf{B u}$ can be attributed to the decrease in electronegativity of the selenium atom as compared to the electronegativity of the sulfur atom. ${ }^{29}$ The small decrease in the ${ }^{1} J_{\text {Se-P }}$ of $\mathbf{2 S e - T h -}{ }^{t} \mathbf{B u}$ as compared to $\mathbf{1 S e - T h}{ }^{i} \mathbf{P r}$ demonstrates the better $\sigma$-donating ability of the tert-butyl groups to transfer electron density to the phosphorus atom. The ${ }^{77} \mathrm{Se}\left\{{ }^{1} \mathrm{H}\right\}$ NMR chemical resonances for 1Se-Th- ${ }^{i}$ Pr and 2 Se-Th- ${ }^{t}$ Bu were 6.4 and 90.5 ppm, respectively, however, no observable trend could be determined. The only other ${ }^{77} \mathrm{Se}\left\{{ }^{1} \mathrm{H}\right\}$ chemical resonance reported for a thorium complex containing a Th-Se bond is $\mathrm{Th}\left[\mathrm{Se}_{2} \mathrm{P}(\mathrm{OMe}) \mathrm{Ph}\right]_{4}\left(\delta=222 \mathrm{ppm},{ }^{1} J_{\mathrm{Se}-\mathrm{P}}=580 \mathrm{~Hz}\right) .{ }^{6}$

Table 3. ${ }^{31} \mathrm{P}\left\{{ }^{1} \mathrm{H}\right\}$ and ${ }^{77} \mathrm{Se}\left\{{ }^{1} \mathrm{H}\right\}$ NMR data for complexes (1S-Th $\left.{ }^{i} \mathbf{P r}\right)-\left(\mathbf{2 S e}-\mathbf{T h}-{ }^{\boldsymbol{t}} \mathbf{B u}\right)$

\begin{tabular}{|c|c|c|}
\hline & ${ }^{31} \mathbf{P}\left\{{ }^{1} \mathbf{H}\right\}(\delta)$ & ${ }^{77} \operatorname{Se}\left\{{ }^{1} \mathbf{H}\right\}(\delta)$ \\
\hline $\operatorname{Th}\left(\mathrm{S}_{2} \mathrm{P}^{i} \operatorname{Pr}_{2}\right)_{4}\left(\mathbf{1 S}-\mathbf{T h}-{ }^{i} \mathbf{P r}\right)$ & 84.9 & --- \\
\hline $\mathrm{Th}\left(\mathrm{S}_{2} \mathrm{P}^{t} \mathrm{Bu}_{2}\right)_{4}\left(\mathbf{2 S}-\mathbf{T h}-{ }^{t} \mathbf{B u}\right)$ & 99.1 & --- \\
\hline $\mathrm{Th}\left(\mathrm{Se}_{2} \mathrm{P}^{i} \operatorname{Pr}_{2}\right)_{4}\left(\mathbf{1 S e}-\mathbf{T h}-{ }^{i} \mathbf{P r}\right)$ & $57.6\left(\mathrm{~s}+\mathrm{d}\right.$ satellites, $\left.{ }^{1} J_{\mathrm{Se}-\mathrm{P}}=500 \mathrm{~Hz}\right)$ & $6.4\left(\mathrm{~d},{ }^{1} J_{\mathrm{P}-\mathrm{Se}}=500 \mathrm{~Hz}\right)$ \\
\hline $\mathrm{Th}\left(\mathrm{Se}_{2} \mathrm{P}^{t} \mathrm{Bu}_{2}\right)_{4}\left(\mathbf{2 S e}-\mathbf{T h}-{ }^{t} \mathbf{B u}\right)$ & $78.3\left(\mathrm{~s}+\mathrm{d}\right.$ satellites, $\left.{ }^{1} J_{\mathrm{Se}-\mathrm{P}}=491 \mathrm{~Hz}\right)$ & $90.5\left(\mathrm{~d},{ }^{1} J_{\mathrm{P}-\mathrm{Se}}=491 \mathrm{~Hz}\right)$ \\
\hline
\end{tabular}


Table 4. ${ }^{1} \mathrm{H}$ and ${ }^{31} \mathrm{P}\left\{{ }^{1} \mathrm{H}\right\}$ NMR data for complexes (1S-U- $\left.{ }^{i} \mathbf{P r}\right)-\left(\mathbf{2 S e}-\mathbf{U}-{ }^{\boldsymbol{t}} \mathbf{B u}\right)$

\begin{tabular}{|c|c|c|}
\hline & ${ }^{1} \mathbf{H}(\delta)$ & ${ }^{31} \mathbf{P}\left\{{ }^{1} \mathbf{H}\right\}(\delta)$ \\
\hline $\mathrm{U}\left(\mathrm{S}_{2} \mathrm{P}^{i} \mathrm{Pr}_{2}\right)_{4}\left(\mathbf{1 S}-\mathbf{U}-{ }^{i} \mathbf{P r}\right)$ & $8.00\left(\mathrm{CH}\left(\mathrm{CH}_{3}\right)_{2}\right), 4.20\left(\mathrm{CH}\left(\mathrm{CH}_{3}\right)_{2}\right)$ & -436.3 \\
\hline $\mathrm{U}\left(\mathrm{S}_{2} \mathrm{P}^{t} \mathrm{Bu}_{2}\right)_{4}\left(\mathbf{2 S}-\mathbf{U}_{-}{ }^{t} \mathbf{B u}\right)$ & -11.90 & -467.2 \\
\hline $\mathrm{U}\left(\mathrm{Se}_{2} \mathrm{P}^{i} \mathrm{Pr}_{2}\right)_{3} \mathrm{Cl}\left(\mathbf{3 S e}-\mathbf{U}^{i \mathbf{P r}}-\mathbf{C l}\right)$ & $7.33\left(\mathrm{CH}\left(\mathrm{CH}_{3}\right)_{2}\right), 4.36\left(\mathrm{CH}\left(\mathrm{CH}_{3}\right)_{2}\right)$ & -610.2 \\
\hline $\mathrm{U}\left(\mathrm{Se}_{2} \mathrm{P}^{t} \mathrm{Bu}_{2}\right)_{3} \mathrm{Cl}\left(\mathbf{4 S e}-\mathbf{U}^{t \mathrm{Bu}}-\mathbf{C l}\right)$ & -2.31 & -690.0 \\
\hline $\mathrm{U}\left(\mathrm{Se}_{2} \mathrm{P}^{i} \operatorname{Pr}_{2}\right)_{4}\left(\mathbf{1 S e}-\mathbf{U}^{i} \mathbf{P r}\right)$ & $7.34\left(\mathrm{CH}\left(\mathrm{CH}_{3}\right)_{2}\right), 4.37\left(\mathrm{CH}\left(\mathrm{CH}_{3}\right)_{2}\right)$ & -618.0 \\
\hline $\mathrm{U}\left(\mathrm{Se}_{2} \mathrm{P}^{t} \mathrm{Bu}_{2}\right)_{4}\left(\mathbf{2 S e}-\mathbf{U}^{t}{ }^{t} \mathbf{B u}\right)$ & 2.74 & -473.0 \\
\hline
\end{tabular}

The ${ }^{1} \mathrm{H}$ and ${ }^{31} \mathrm{P}\left\{{ }^{1} \mathrm{H}\right\}$ NMR spectroscopy of complexes $\left(\mathbf{1 S}-\mathbf{U}-{ }^{i} \mathbf{P r}\right)-\left(\mathbf{2 S e}-\mathbf{U}-{ }^{\boldsymbol{t}} \mathbf{B u}\right)$ are listed in Table 4. The ${ }^{1} \mathrm{H}$ spectra for complexes (1S-U- $\left.{ }^{\boldsymbol{i}} \mathbf{P r}\right)-\left(\mathbf{2 S e}-\mathbf{U}^{\boldsymbol{t}}{ }^{\mathbf{B}} \mathbf{B}\right)$ all exhibited paramagnetically shifted resonances for their respective alkyl groups. The ${ }^{31} \mathrm{P}\left\{{ }^{1} \mathrm{H}\right\}$ resonances were also paramagnetically shifted. One noticeable feature in the ${ }^{31} \mathrm{P}\left\{{ }^{1} \mathrm{H}\right\}$ is the downfield shift in the chemical resonance of the homoleptic of $\mathrm{U}\left[\mathrm{S}_{2} \mathrm{P}^{i} \mathrm{Pr}_{2}\right]_{4}$ and $\mathrm{U}\left[\mathrm{S}_{2} \mathrm{P}^{t} \mathrm{Bu}_{2}\right]_{4}$ as compared to complexes 7-10. The downfield shift can be explained by the more electronegative sulfur atom bound to the phosphorus as previously mentioned with the homoleptic thorium complexes. The IR spectroscopy experiments were conducted for complexes (1S-Th- $\left.{ }^{i} \mathbf{P r}\right)-\left(\mathbf{2 S e}-\mathbf{T h}-{ }^{\mathbf{t}} \mathbf{B u}\right)$ and selected results are tabulated in Table S3.

The solid-state structures of $\left(\mathbf{1 S - T h}-{ }^{i} \mathbf{P r}\right)-\left(\mathbf{2 S e}-\mathbf{T h}{ }^{-}{ }^{\mathbf{B} u}\right)$ were determined through X-ray crystallography analysis. Complexes $\left(\mathbf{1 S - T h}-{ }^{i} \mathbf{P r}\right)-\left(\mathbf{2 S e}-\mathbf{T h}-{ }^{t} \mathbf{B u}\right)$ were homoleptic with pseudo $\mathrm{C}_{4 v}$ symmetry (Figure 1). Each chalcogenide atom is bonded to the thorium metal center given rise to a coordination number of eight for complexes $\left(\mathbf{1 S - T h}-{ }^{i} \mathbf{P r}\right)-\left(\mathbf{2 S e}-\mathbf{T h}-{ }^{t} \mathbf{B u}\right)$ and the geometry can be best described at a triangular dodecahedron for all four complexes. ${ }^{30}$ Each $\left[\mathrm{E}_{2} \mathrm{PR}_{2}\right]^{-}\left(\mathrm{E}=\mathrm{S}, \mathrm{Se} ; \mathrm{R}={ }^{i} \mathrm{Pr},{ }^{t} \mathrm{Bu}\right)$ ligand is nearly coplanar with the other with a Se1-Se2-Se3- 
Se4 dihedral angle of $2.10^{\circ}$ and almost orthogonal to the other two with a Se2-Th1-Se4-P2 dihedral angle of $96.90^{\circ}$. No observable Th-P bond was found in complexes (1S-Th- $\left.{ }^{i} \mathbf{P r}\right)-(\mathbf{2 S e}-$ Th- $\left.{ }^{t} \mathrm{Bu}\right)$. A slight deviation in the Th-E bond distances was observed for each $\left[\mathrm{E}_{2} \mathrm{PR}_{2}\right]^{-}$ligand, one short and one long. This feature was observed for other homoleptic thorium dichalogenide complexes, $\mathrm{Th}\left[\mathrm{S}_{2} \mathrm{P}\left(4-\mathrm{MeOC}_{6} \mathrm{H}_{4}\right)(\mathrm{OMe})\right]_{4}$ and $\mathrm{Th}\left[\mathrm{Se}_{2} \mathrm{P}\left(\mathrm{C}_{6} \mathrm{H}_{5}\right)(\mathrm{OMe})\right]_{4}{ }^{6}$ The Th-E and E-Th-E bond distances and angles are listed in Table 5 .

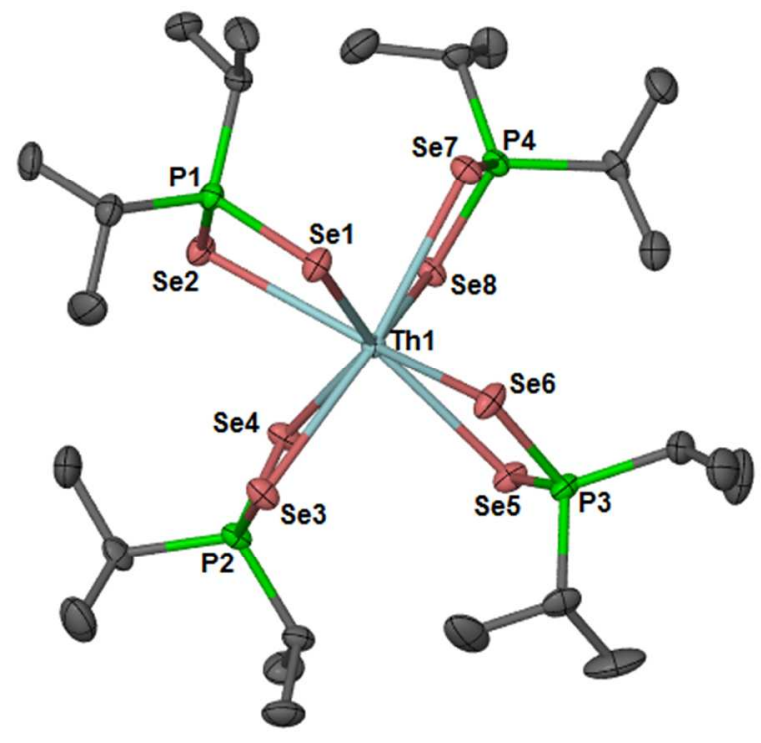

Figure 1. Complex 3 is shown as a representative thermal ellipsoid plot of homoleptic $\mathrm{Th}\left[\mathrm{E}_{2} \mathrm{P}^{i} \mathrm{Pr}_{2}\right]_{4}, \mathrm{E}=\mathrm{S}$, Se, complexes. Thermal ellipsoids at $50 \%$ and hydrogens omitted for clarity.

Table 5. Selected bond distances $(\AA)$ and angles $\left({ }^{\circ}\right)$ for $\mathrm{Th}\left[\mathrm{S}_{2} \mathrm{P}^{i} \mathrm{Pr}_{2}\right]_{4}\left(\mathbf{1 S}-\mathbf{T h}{ }^{i} \mathbf{P r}\right), \mathrm{Th}\left[\mathrm{S}_{2} \mathrm{P}^{t} \mathrm{Bu}_{2}\right]_{4}$ (2S-Th- $\left.{ }^{t} \mathbf{B u}\right), \mathrm{Th}\left[\mathrm{Se}_{2} \mathrm{P}^{i} \mathrm{Pr}_{2}\right]_{4}\left(\mathbf{1 S e}-\mathbf{T h}-{ }^{i} \mathrm{Pr}\right)$, and $\mathrm{Th}\left[\mathrm{Se}_{2} \mathrm{P}^{t} \mathrm{Bu}_{2}\right]_{4}\left(\mathbf{2 S e}-\mathbf{T h}-{ }^{t} \mathbf{B u}\right)$.

1Se-Th- ${ }^{i} \mathbf{P r}$

Th1-Se1

Th1-Se2

Th1-Se3

Th1-Se4

Th1-Se5

Th1-Se6
3.0576(7)

$3.0107(6)$

3.0023(6)

$3.0477(7)$

$3.0379(6)$

3.0492(7)
2Se-Th- ${ }^{t} \mathrm{Bu}$

$3.0636(6)$

$3.0612(7)$

$3.0291(6)$

$3.0653(7)$
2.8882(5)

6
7
8

Th1-S6

2.9307(6)

$2.915(2)$
$2.940(2)$

- 
The average Th-S bond distance was $2.9075(5) \AA$ and 2.932(2) $\AA$ for complexes $\mathbf{1 S - T h}-{ }^{i} \mathbf{P r}$ and 2S-Th- ${ }^{t} \mathbf{B u}$, respectively, while the average Th-Se bond distance was 3.0377(6) $\AA$ and 3.0548(8)

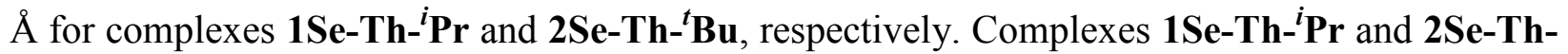
${ }^{t}$ Bu represent only the second and third structurally characterized homoleptic complexes containing a Th-Se linkage and compares well to the first homoleptic thorium complex with selenium atoms, $\operatorname{Th}\left[\mathrm{Se}_{2} \mathrm{P}\left(\mathrm{C}_{6} \mathrm{H}_{5}\right)(\mathrm{OMe})\right]_{4}$, the average Th-Se distance of 3.0261(4) $\AA^{6}{ }^{6}$ These bond distances are longer than those in $\left(1,2,4-{ }^{t} \mathrm{Bu}_{3} \mathrm{C}_{5} \mathrm{H}_{2}\right)_{2} \mathrm{Th}(\mathrm{SePh})_{2}$ and $(1,2,4-$ $\left.{ }^{t} \mathrm{Bu}_{3} \mathrm{C}_{5} \mathrm{H}_{2}\right) \mathrm{Th}(\mathrm{SePh})_{3}\left(\right.$ bipy) reported average Th-Se distances of $2.938(8)^{31}$ and $2.877 \AA,{ }^{32}$ respectively. The elongated Th-Se bonds in the homoleptic compounds arise from the negative charge spread over two donor atoms.

The homoleptic U(IV) complexes (1S-U- ${ }^{i} \mathbf{P r}, \mathbf{1 S e}-{ }^{\mathbf{U}}-{ }^{i} \mathbf{P r}$ and $\left.\mathbf{2 S e - U -}{ }^{t} \mathbf{B u}\right)$ are isostructural with the thorium analogs (Figure 2) with selected bond distances and angles listed in Table 6. The IR spectroscopy experiments were conducted for complexes $\mathbf{1 S - U}-{ }^{i} \mathbf{P r}, \mathbf{2 S}-\mathbf{U}-{ }^{t} \mathbf{B u}, \mathbf{1 S e}-\mathbf{U}-$ ${ }^{i} \mathbf{P r}$, and $\mathbf{2 S e}-\mathbf{U}-{ }^{t} \mathbf{B u}$ and selected results are tabulated in Table S4. 


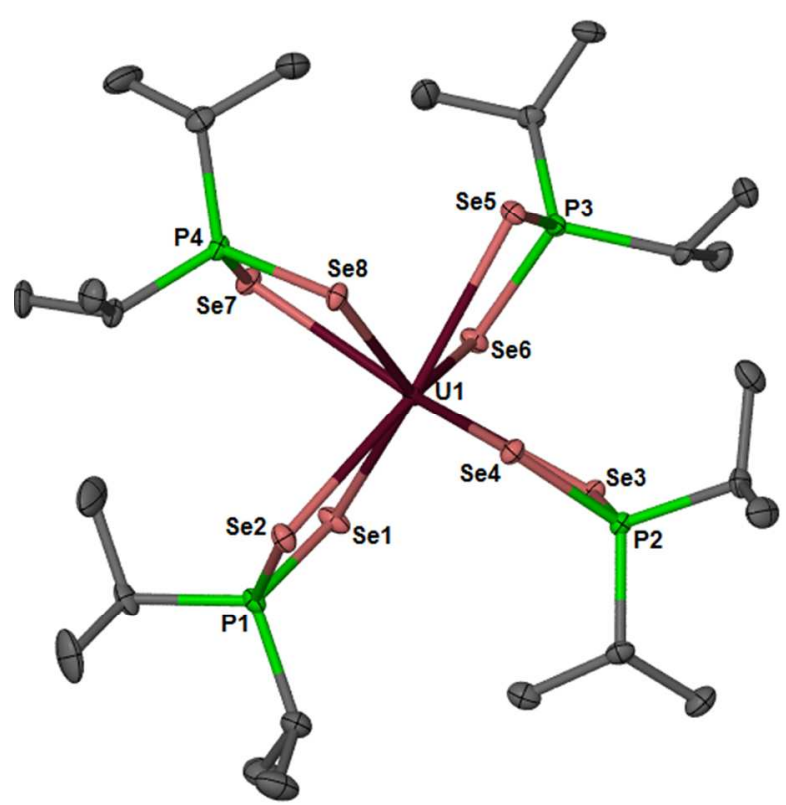

Figure 2. Complex $\mathbf{1 S e}-\mathbf{U}-{ }^{i} \mathbf{P r}$ is shown as a representative thermal ellipsoid plot of homoleptic $\mathrm{U}\left[\mathrm{E}_{2} \mathrm{P}^{i} \mathrm{Pr}_{2}\right]_{4}, \mathrm{E}=\mathrm{S}$, Se, complexes. Thermal ellipsoids at $50 \%$ and hydrogens omitted for clarity.

Table 6. Selected bond distances $(\AA)$ and angles $\left(^{\circ}\right)$ for $U\left[\mathrm{~S}_{2} \mathrm{P}^{i} \operatorname{Pr}_{2}\right]_{4}\left(\mathbf{1 S}-\mathbf{U}-{ }^{i} \mathbf{P r}\right), \mathrm{U}\left[\mathrm{Se}_{2} \mathrm{P}^{i} \operatorname{Pr}_{2}\right]_{4}$ (1Se-U- $\left.{ }^{i} \mathbf{P r}\right)$, and $\mathrm{U}\left[\mathrm{Se}_{2} \mathrm{P}^{t} \mathrm{Bu}_{2}\right]_{4}\left(2 \mathrm{Se}-\mathbf{U}_{-}{ }^{t} \mathbf{B u}\right)$.

\begin{tabular}{|c|c|c|c|c|}
\hline & 1S-U- ${ }^{i} \mathbf{P r}$ & & 1Se-U- ${ }^{i} \mathbf{P r}$ & $2 \mathrm{Se}-\mathrm{U}-{ }^{t} \mathrm{Bu}$ \\
\hline U1-S1 & $2.885(3)$ & U1-Se1 & $3.0076(4)$ & $2.9973(10)$ \\
\hline U1-S2 & $2.809(3)$ & U1-Se2 & $2.9859(4)$ & $3.0204(11)$ \\
\hline U1-S3 & $2.809(3)$ & U1-Se3 & $3.0263(4)$ & $3.0318(11)$ \\
\hline U1-S4 & $2.879(4)$ & U1-Se4 & $3.0477(7)$ & $2.9601(10)$ \\
\hline U1-S5 & $2.876(3)$ & U1-Se5 & $2.9362(4)$ & - \\
\hline U1-S6 & $2.840(3)$ & U1-Se6 & $3.0192(4)$ & - \\
\hline U1-S7 & $2.843(4)$ & U1-Se7 & $2.9343(4)$ & - \\
\hline U1-S8 & $2.842(4)$ & U1-Se8 & $3.0092(4)$ & - \\
\hline S1-U1-S2 & $70.49(9)$ & Se1-U1-Se2 & $71.561(15)$ & $69.90(3)$ \\
\hline S3-U1-S4 & $70.37(10)$ & Se3-U1-Se4 & 71.017(10) & $70.10(3)$ \\
\hline S5-U1-S6 & $70.03(10)$ & Se5-U1-Se6 & $72.575(10)$ & - \\
\hline S7-U1-S8 & $70.28(11)$ & Se7-U1-Se8 & $71.302(11)$ & - \\
\hline S1-P1-S2 & 109.01(19) & Se1-P1-Se2 & $106.99(4)$ & $104.44(11)$ \\
\hline S3-P2-S4 & 109.1(2) & $\mathrm{Se} 3-\mathrm{P} 2-\mathrm{Se} 4$ & 106.99(4) & $104.06(11)$ \\
\hline S5-P3-S6 & $109.1(2)$ & Se5-P3-Se6 & $108.24(4)$ & - \\
\hline S7-P4-S8 & $104.6(3)$ & Se7-P4-Se8 & $108.70(4)$ & - \\
\hline
\end{tabular}


Complexes 3Se- $\mathbf{U}^{i \mathbf{P r}}-\mathbf{C l}$ and $\mathbf{4 S e}-\mathbf{U}^{t \mathbf{B u}}-\mathbf{C l}$ both contain one chloride ligand, however, the remaining $\left[\mathrm{Se}_{2} \mathrm{PR}_{2}\right]^{-}\left(\mathrm{R}={ }^{i} \mathrm{Pr},{ }^{t} \mathrm{Bu}\right)$ ligands adopt very different geometries around the uranium metal center (Figures 3 and 4). For complex 3Se-U ${ }^{i \mathbf{P r}}-\mathbf{C l}$, two of the $\left[\mathrm{Se}_{2} \mathrm{P}^{i} \operatorname{Pr}_{2}\right]^{-}$ligands are nearly coplanar with each other with Se3-Se4-Se6-Se5 dihedral angle of $2.42^{\circ}$. The third $\left[\operatorname{Se}_{2} \mathrm{P}^{i} \operatorname{Pr}_{2}\right]^{-}$ ligand is almost orthogonal to the other two with a Se6-U1-Se2-P1 dihedral angle of $87.02^{\circ}$. This geometric arrangement is the likely result of minimizing the unfavorable interactions between the isopropyl groups on the phosphorus atoms. Another unique feature exclusive to $3 \mathbf{S e}-\mathbf{U}^{i \mathbf{P r}}-\mathbf{C l}$ is the U1-Se1 and U1-Se2 bond distances (Table 7). The U1-Se1 bond length is 2.9003(5) $\AA$ while the U1-Se2 3.0038(5) $\AA$. As in the thorium complexes, these are elongated with respect to other mononuclear U(IV) complexes such as 2.7897(7) and 2.8597(8) $\AA$ in $[\mathrm{K}(18$-crown6)] $\left[\left(\mathrm{R}_{2} \mathrm{~N}\right)_{3} \mathrm{U}\left(\eta^{2}-\mathrm{Se}_{2}\right)\right], \mathrm{R}=\mathrm{SiMe}_{3},{ }^{33}$ or $2.8432(7) \AA$ in $\left(\mathrm{C}_{5} \mathrm{Me}_{5}\right)_{2} \mathrm{U}(\mathrm{SePh})_{2} \cdot{ }^{34}$ In complex $\mathbf{4 S e}-\mathbf{U}^{t \mathrm{Bu}}-$ Cl, the Se3-Se4-Se6-Se5 dihedral angle of $68.39^{\circ}$ and a U1-Se1 and U1-Se2 bond distances of

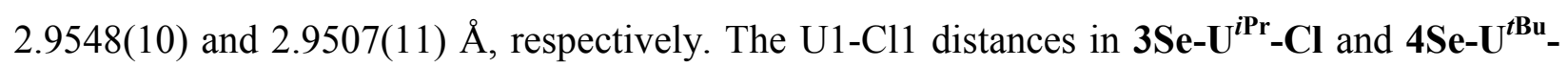
Cl are 2.5632(12) and 2.587(2) $\AA$, respectively and shorter than $2.637 \AA$ in $\left(\mathrm{C}_{5} \mathrm{Me}{ }_{4} \mathrm{H}\right)_{3} \mathrm{UCl},{ }^{35}$ $2.6099(15) \AA$ in $\left(\mathrm{C}_{5} \mathrm{Me}_{5}\right)_{2} \mathrm{U}(\mathrm{Cl})\left(\mathrm{NPh}_{2}\right),{ }^{36}$ and $2.6783(10) \AA$ in $\left\{\mathrm{U}\left[\mathrm{MeC}(\mathrm{NCy})_{2}\right]_{3} \mathrm{Cl}\right\} .{ }^{37}$

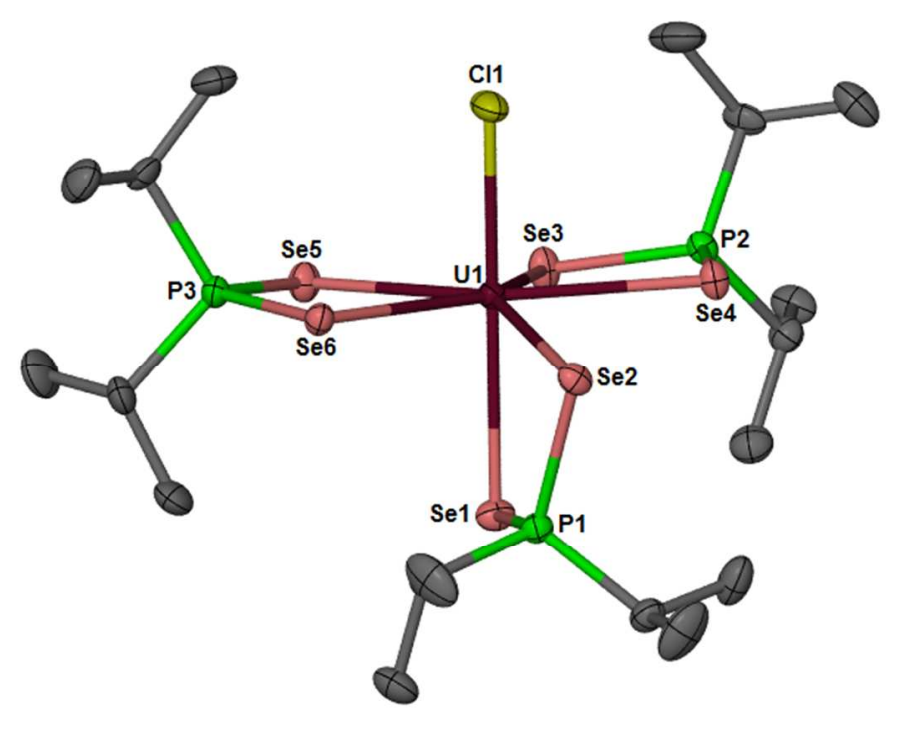


Figure 3. Thermal ellipsoid plot of $U\left(\mathrm{Se}_{2} \mathrm{P}^{i} \mathrm{Pr}_{2}\right)_{3} \mathrm{Cl}\left(\mathbf{3 S e}-\mathbf{U}^{i \mathbf{P r}}-\mathbf{C l}\right)$. Thermal ellipsoids at $50 \%$ and hydrogens omitted for clarity.

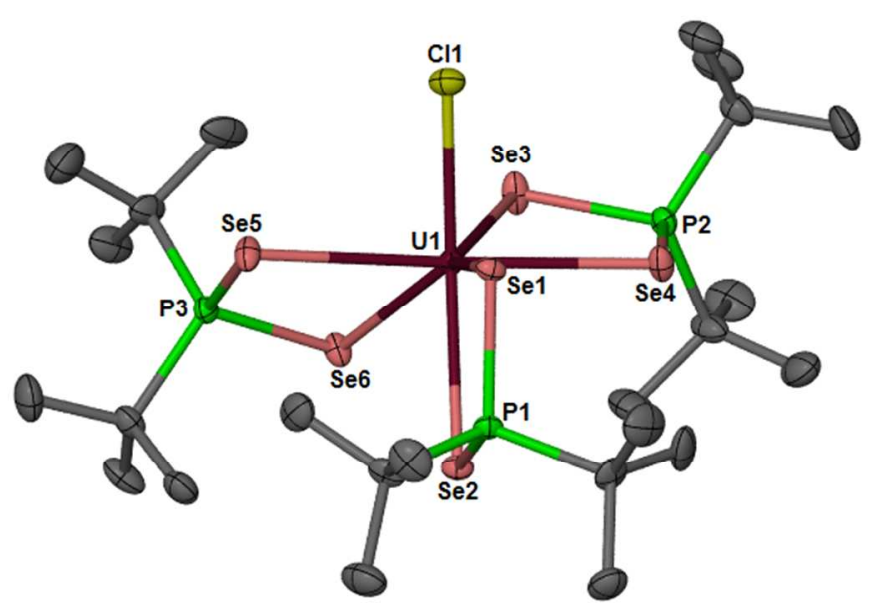

Figure 4. Thermal ellipsoid plot of $\mathrm{U}\left(\mathrm{Se}_{2} \mathrm{P}^{t} \mathrm{Bu}_{2}\right)_{3} \mathrm{Cl}\left(\mathbf{4 S e}-\mathbf{U}^{t \mathbf{B u}}-\mathbf{C l}\right)$. Thermal ellipsoids at $50 \%$ and hydrogens omitted for clarity.

Table 7. Selected bond distances $(\AA)$ and angles $\left({ }^{\circ}\right)$ for $\mathrm{U}\left(\mathrm{Se}_{2} \mathrm{P}^{i} \mathrm{Pr}_{2}\right)_{3} \mathrm{Cl}\left(\mathbf{3 S e}-\mathbf{U}^{i \mathbf{P r}}-\mathbf{C l}\right)$ and $\mathrm{U}\left(\mathrm{Se}_{2} \mathrm{P}^{t} \mathrm{Bu}_{2}\right)_{3} \mathrm{Cl}\left(\mathbf{4 S e}-\mathbf{U}^{t \mathrm{Bu}}-\mathbf{C l}\right)$.

\begin{tabular}{ccc}
\hline & 3Se-U $^{\text {iPr }}-\mathbf{C l}$ & 4Se-U $^{\text {tBu}}-\mathbf{C l}$ \\
U1-Cl1 & $2.5632(12)$ & $2.587(2)$ \\
U1-Se1 & $2.9003(5)$ & $2.9548(10)$ \\
U1-Se2 & $3.0038(5)$ & $2.9507(11)$ \\
U1-Se3 & $2.9228(5)$ & $2.9773(10)$ \\
U1-Se4 & $2.9146(5)$ & $2.9136(11)$ \\
U1-Se5 & $2.9599(5)$ & $2.9329(11)$ \\
U1-Se6 & $2.9410(5)$ & $2.9483(10)$ \\
Cl1-U1-Se1 & $173.09(3)$ & $77.41(6)$ \\
Cl1-U1-Se2 & $98.18(3)$ & $149.67(6)$ \\
Se1-U1-Se2 & $75.374(13)$ & $69.90(3)$ \\
Se3-U1-Se4 & $74.228(14)$ & $72.51(3)$ \\
Se5-U1-Se6 & $73.479(14)$ & $73.35(3)$ \\
Se1-P1-Se2 & $111.54(5)$ & $106.34(11)$ \\
Se3-P2-Se4 & $108.18(5)$ & $106.55(11)$ \\
Se5-P3-Se6 & $108.58(5)$ & $107.53(11)$ \\
\hline
\end{tabular}

The reactivity of $\mathbf{2 S e}-\mathbf{T h}-{ }^{\mathbf{t}} \mathbf{B u}$ was investigated through transmetalation reactions with various copper salts $\left(\mathrm{CuCl}_{2}, \mathrm{CuBr}\right.$, and $\left.\mathrm{CuI}\right)$. Interestingly, based on the ${ }^{1} \mathrm{H} \mathrm{NMR}$ spectrum, only starting material was observed after letting the reaction stir for $24 \mathrm{~h}$ at room temperature. In an attempt to 
explain the dearth of reactivity exhibited by $\mathbf{2 S e - T h -}{ }^{t} \mathbf{B u}$, we compared the averaged difference in the An-Se bond $(\Delta,[\mathrm{Th}-\mathrm{Se}]-[\mathrm{U}-\mathrm{Se}])$ in $\mathbf{2 S e - T h}{ }^{t} \mathbf{B u}$ and $\mathbf{2 S e - U}-{ }^{\boldsymbol{t}} \mathbf{B u}$ to the decrease in ionic radii from an eight coordinate $\mathrm{Th}^{4+}(1.190 \AA)$ to $\mathrm{U}^{4+}(1.140 \AA) .{ }^{38}$ The difference in ionic radii for an eight coordinate $\mathrm{Th}^{4+}$ to $\mathrm{U}^{4+}$ is c.a. $0.050 \AA$ and the $\Delta$ value we obtained was $0.052 \AA$ showing there is no difference in the An-Se bond distance between Th and U. A closer comparison of the Th-Se and U-Se bond ranges between 2 Se-Th- ${ }^{t} \mathbf{B u}$ and $2 \mathbf{S e}-\mathbf{U}_{-}{ }^{t} \mathbf{B u}$ resulted in a greater range for $\mathrm{U}\left[\mathrm{Se}_{2} \mathrm{P}^{t} \mathrm{Bu}_{2}\right]_{4}$. The U-Se bond lengths span from 2.9973(10) to 3.0318(11) $\AA$ and the Th-Se bond lengths from 3.0291(6) to 3.0653(7) A. We also compared the average U-Se bond length of $\left[\mathrm{U}\left(\mathrm{Se}_{2} \mathrm{PPh}_{2}\right)_{4}\right]^{5}$ to the average U-Se bond lengths in $\mathbf{1 S e -} \mathbf{U}^{i}{ }^{i} \mathbf{P r}$ and $\mathbf{2 S e}-\mathbf{U}^{\boldsymbol{t}}{ }^{\mathbf{B u}}$. We found that replacement of the phenyl ligand for iso-propyl and tert-butyl ligands resulted in an increase of the U-Se bond length by $0.0279 \AA$ and $0.0345 \AA$, respectively. The increase in USe bond distances for $\mathbf{1 S e -} \mathbf{U}_{-}^{\boldsymbol{i}} \mathbf{P r}$ and $\mathbf{2 S e}-\mathbf{U}_{-}{ }^{\boldsymbol{t}} \mathbf{B u}$ can be attributed to the steric repulsions

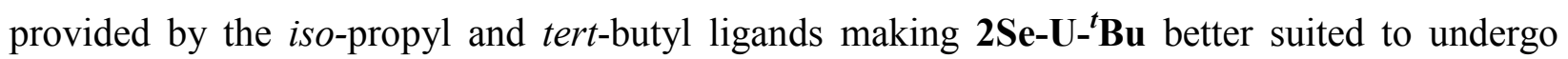
transmetalation reactions.

Presumably due to steric considerations of the dithio- and diselenophosphinate complexes of

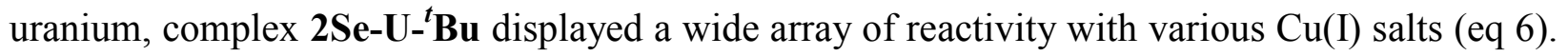
The transmetalation reactions with $\mathrm{Cu}(\mathrm{I})$ halides resulted in dark red color change with concomitant formation of $\left[\mathrm{Cu}\left(\mathrm{Se}_{2} \mathrm{P}^{t} \mathrm{Bu}_{2}\right)\right]_{4}, \quad \mathbf{8 S e}-\mathbf{C u}-{ }^{t} \mathbf{B u}$. Complex $\mathbf{8 S e}-\mathbf{C u}-{ }^{t} \mathbf{B u}$ was independently synthesized from the stoichiometric reaction between $\left[\mathrm{Cu}(\mathrm{NCMe})_{4}\right]\left[\mathrm{PF}_{6}\right]$ and $\mathrm{K}\left(\mathrm{Se}_{2} \mathrm{P}^{t} \mathrm{Bu}_{2}\right)$ in acetonitrile. The ${ }^{1} \mathrm{H}$ NMR spectrum showed one doublet centered at $1.51 \mathrm{ppm}$ with ${ }^{3} J_{\mathrm{P}-\mathrm{H}}=17.0 \mathrm{~Hz}$ representing the tert-butyl protons. The ${ }^{31} \mathrm{P}\left\{{ }^{1} \mathrm{H}\right\}$ spectrum displayed a singlet at $90.6 \mathrm{ppm}$ with ${ }^{77} \mathrm{Se}$ satellites and ${ }^{1} J_{\mathrm{Se}-\mathrm{P}}=533 \mathrm{~Hz}$. The solid-state structure of $\mathbf{8 S e - C u}-$ ${ }^{t} \mathbf{B u}$ was solved using X-ray quality crystals grown from a concentrated toluene/hexanes mixture 
at $-20{ }^{\circ} \mathrm{C}$ (Figure 5). Complex $\mathbf{8 S e}-\mathbf{C u}-{ }^{t} \mathbf{B u}$ is a tetranuclear cluster with Cu1-Se1 bond distance of 2.3862(14) $\AA$ and $\mathrm{Cu} 2-\mathrm{Se} 4$ bond distance of 2.3986(14) $\AA$ (Table 8). Each $\left[\mathrm{Se}_{2} \mathrm{P}^{t} \mathrm{Bu}_{2}\right]^{-}$ligand is bridging rather than chelating. In one $\left[\mathrm{Se}_{2} \mathrm{P}^{t} \mathrm{Bu}_{2}\right]^{-}$motif, each selenium atom is bound to a phosphorus atom and two different copper atoms, while one selenium atom is bound to one copper metal and a phosphorus atom in the second $\left[\mathrm{Se}_{2} \mathrm{P}^{t} \mathrm{Bu}_{2}\right]^{-}$ligand. Therefore, each copper center is coordinated by three selenium atoms resulting in a cubane structure. The $\mathrm{Se}-\mathrm{Cu}-\mathrm{Se}$ angles span from $126.54(5)$ to $104.55(5)^{\circ}$ placing the copper atoms a distorted trigonal planar geometry. A similar structure, $\left[\mathrm{Cu}\left(\mathrm{Se}_{2} \mathrm{P}^{i} \mathrm{Pr}_{2}\right)\right]_{4}$, has been previous characterized. ${ }^{39}$

To rule out the possibility of a redox reaction occurring between uranium and copper, a non-

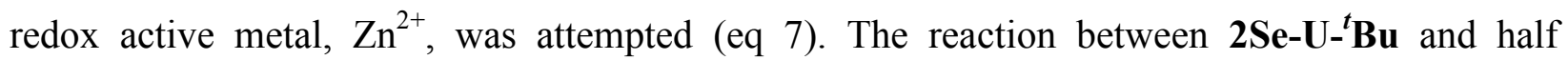
equivalent of $\mathrm{ZnCl}_{2}$ resulted in a red solution and the ${ }^{1} \mathrm{H}$ and ${ }^{31} \mathrm{P}\left\{{ }^{1} \mathrm{H}\right\}$ NMR spectra showed the formation of $\mathbf{4 S e}-\mathbf{U}^{\mathrm{tBu}}-\mathbf{C l}$ as well as a second product (Figure S9). The product was identified as $\mathrm{Zn}\left(\mathrm{Se}_{2} \mathrm{P}^{t} \mathrm{Bu}_{2}\right)_{2}$ and was independently synthesized from the reaction between $\mathrm{ZnCl}_{2}$ and two equivalents of $\mathrm{KSe}_{2} \mathrm{P}^{t} \mathrm{Bu}_{2}$ (See Supporting Information). ${ }^{1} \mathrm{H}$ and ${ }^{31} \mathrm{P}\left\{{ }^{1} \mathrm{H}\right\} \mathrm{NMR}$ spectroscopy as well as X-ray crystallography confirmed the identity of the $\mathrm{Zn}\left(\mathrm{Se}_{2} \mathrm{P}^{\mathrm{t}} \mathrm{Bu}_{2}\right)_{2}$ product. An additional transmetalation reaction was attempted using $\mathrm{Hg}_{2} \mathrm{~F}_{2}$ to yield the mono-fluoride complex, 5Se$\mathbf{U}^{\mathbf{t B u}}-\mathbf{F}$ (eq 7). To disseminate the mercury byproduct, the independent stoichiometric reaction between $\mathrm{Hg}_{2} \mathrm{~F}_{2}$ and $\mathrm{KSe}_{2} \mathrm{P}^{\mathrm{t}} \mathrm{Bu}_{2}$ was conducted and ${ }^{1} \mathrm{H}$ and ${ }^{31} \mathrm{P}\left\{{ }^{1} \mathrm{H}\right\}$ NMR spectroscopy matched the resonance in the transmetalation reaction (See Supporting Information). We are still unclear as to the reason for reactivity of $\mathbf{2} \mathbf{S e}-\mathbf{U}-{ }^{t} \mathbf{B u}$ with $\mathrm{Cu}(\mathrm{I}), \mathrm{ZnCl}_{2}$, and $\mathrm{Hg}_{2} \mathrm{~F}_{2}$ salts. It would seem that an redox event involving a $\mathrm{U}(\mathrm{V})$ intermediate can be ruled out on the basis of a successful transmetalation reaction with non-redox active $\mathrm{ZnCl}_{2}$ salt. Given that $\mathbf{2} \mathbf{S e}-\mathbf{T h}-{ }^{\boldsymbol{t}} \mathbf{B u}$ and $\mathbf{2 S e}-\mathbf{U}-{ }^{\boldsymbol{t}} \mathbf{B u}$ have nearly identical actinide-selenium bond distances, we make the argument that because of 


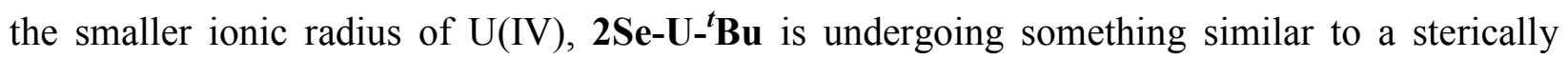
induced transmetalation reaction with $\mathrm{Cu}(\mathrm{I}), \mathrm{ZnCl}_{2}$, and $\mathrm{Hg}_{2} \mathrm{~F}_{2}$ salts to yield the heteroleptic mono-halide complexes to relieve steric strain. This type of sterically induced reactivity is known to f elements, ${ }^{40-44}$ and has been argued before in the coordination chemistry of Cyanex 301 with actinides. ${ }^{45}$

This is not the first time group 11 salts have been used in actinide chemistry. For example, previous reports have used copper and gold salts to oxidize $U\left(\right.$ III) to $U(I V)^{46-49}$ or $U(I V)$ to $\mathrm{U}(\mathrm{V}) .{ }^{50-53}$ Alternatively, the Evans group made use of copper $(\mathrm{CuX})$ and silver $(\mathrm{AgX})$ salts for transmetalation reactions with $\left(\mathrm{C}_{5} \mathrm{Me}_{5}\right)_{2} \mathrm{U}\left(\mathrm{CH}_{3}\right)_{2}$ to yield complexes of the form $\left(\mathrm{C}_{5} \mathrm{Me}_{5}\right)_{2} \mathrm{U}\left(\mathrm{CH}_{3}\right) \mathrm{X}, \mathrm{X}=\mathrm{Br}, \mathrm{I}, \mathrm{OTf}^{54,55} \mathrm{We}$ are unaware of any reports of actinides with mercury salts and this provides a new avenue into synthesizing non-organometallic uranium(IV)-fluoride bonds ${ }^{56-60}$ which are important in the nuclear fuel cycle.

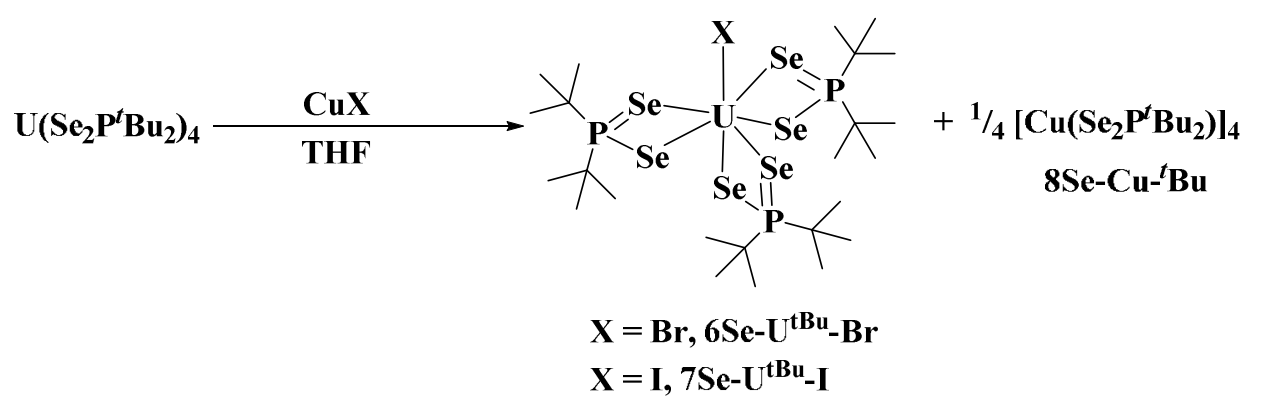




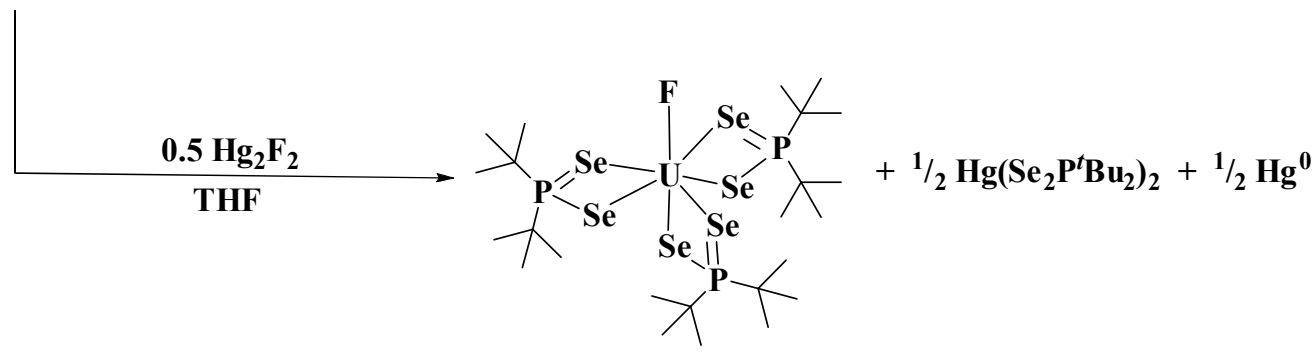

5 Se- ${ }^{\text {tBu}}-F$

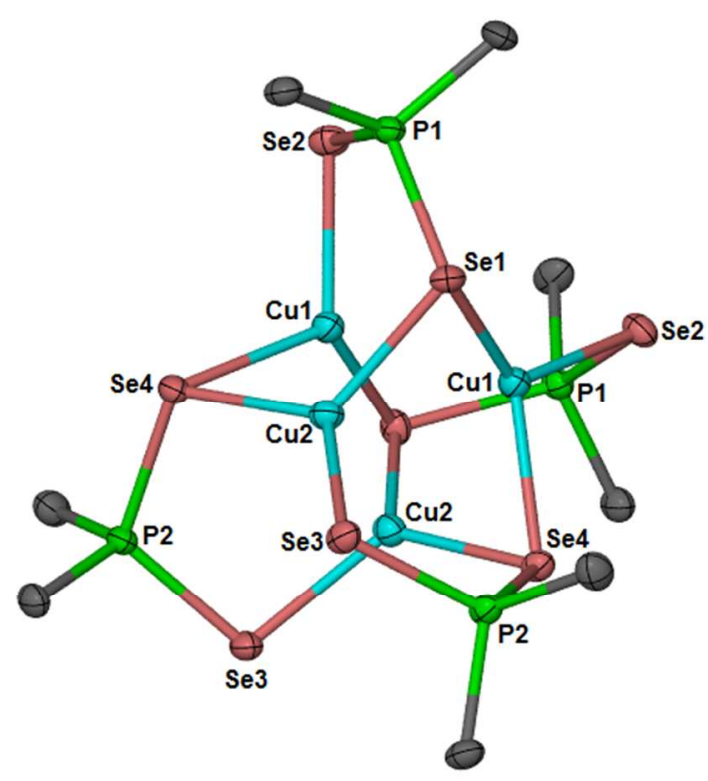

Figure 5. Thermal ellipsoid plot of $\left[\mathrm{Cu}\left(\mathrm{Se}_{2} \mathrm{P}^{t} \mathrm{Bu}_{2}\right)\right]_{4}\left(\mathbf{8 S e}-\mathbf{C u}{ }^{-} \mathbf{B u}\right)$. Thermal ellipsoids at 50\% with tert-butyl carbons and hydrogens omitted for clarity.

Table 8. Selected bond distances $(\AA)$ and angles $\left({ }^{\circ}\right)$ for $\left[\mathrm{Cu}\left(\mathrm{Se}_{2} \mathrm{P}^{t} \mathrm{Bu}_{2}\right)\right]_{4}\left(\mathbf{8 S e}-\mathbf{C u}-{ }^{t} \mathbf{B u}\right)$.

\begin{tabular}{ll}
\hline $\mathrm{Cu} 1-\mathrm{Se} 1$ & $2.3862(14)$ \\
$\mathrm{Cu} 1-\mathrm{Se} 2$ & $2.4233(13)$ \\
$\mathrm{Cu} 1-\mathrm{Se} 4$ & $2.4325(13)$ \\
$\mathrm{Cu} 2-\mathrm{Se} 1$ & $2.4233(13)$ \\
$\mathrm{Cu} 2-\mathrm{Se} 3$ & $2.4216(14)$ \\
$\mathrm{Cu} 2-\mathrm{Se} 4$ & $2.3986(14)$
\end{tabular}


$\mathrm{P} 1-\mathrm{Se} 2$

$\mathrm{P} 2-\mathrm{Se} 3$

$2.227(2)$

P2-Se4

2.9198(13)

2.161(2)

$\mathrm{Se} 1-\mathrm{Cu} 1-\mathrm{Se} 2$

2.232(2)

$\mathrm{Se} 1-\mathrm{Cu} 1-\mathrm{Se} 4$

126.54(5)

$\mathrm{Se} 2-\mathrm{Cu} 1-\mathrm{Se} 4$

$117.82(5)$

$\mathrm{Se} 1-\mathrm{Cu} 2-\mathrm{Se} 3$

$107.85(5)$

$\mathrm{Se} 1-\mathrm{Cu} 2-\mathrm{Se} 4$

$104.55(5)$

123.48(5)

$\mathrm{Se} 3-\mathrm{Cu} 2-\mathrm{Se} 4$

125.21(5)

Se1-P1-Se2

$113.35(10)$

$\mathrm{Se} 3-\mathrm{P} 2-\mathrm{Se} 4$

Complexes 5Se- $\mathbf{U}^{\mathrm{tBu}}-\mathbf{F}, 4 \mathrm{Se}-\mathbf{U}^{\mathrm{tBu}}-\mathbf{C l}, 6 \mathrm{6Se}-\mathbf{U}^{\mathrm{tBu}}-\mathbf{B r}$, and $7 \mathrm{Se}-\mathbf{U}^{\mathrm{tBu}}-\mathbf{I}$ were characterized spectroscopically. Table 9 lists the ${ }^{1} \mathrm{H}$ and ${ }^{31} \mathrm{P}\left\{{ }^{1} \mathrm{H}\right\}$ chemical shifts and the relationship between the chemical shift of the tert-butyl protons and the ancillary $\mathrm{X}$-type ligand $(\mathrm{X}=\mathrm{F}, \mathrm{Cl}, \mathrm{Br}, \mathrm{I})$ can be observed. The chemical shift of the tert-butyl protons is more upfield with substitution of a stronger $\pi$-donor halide ligand $(\mathrm{F}>\mathrm{Cl}>\mathrm{Br}>\mathrm{I})$. This effect can be explained through the stronger shielding experienced by the tert-butyl protons as a result of more electron density at the uranium center (i.e. the stronger $\pi$-donor, the more electron density at the uranium center). A similar feature is observed the ${ }^{31} \mathrm{P}\left\{{ }^{1} \mathrm{H}\right\}$ resonances for the same reason. Similar trends have been noted for various $\mathrm{U}(\mathrm{III})^{61}$ and $\mathrm{U}(\mathrm{IV})^{36,62,63}$ complexes.

Table 9. ${ }^{1} \mathrm{H}{ }^{31} \mathrm{P}\left\{{ }^{1} \mathrm{H}\right\}$ and NMR data for complexes $\mathbf{5 S e}-\mathbf{U}^{\mathrm{tBu}}-\mathbf{F}, \mathbf{4 S e}-\mathbf{U}^{\mathrm{tBu}}-\mathbf{C l}, \mathbf{6 S e}-\mathbf{U}^{\mathbf{t B u}}-$ Br, and 7Se- $\mathbf{U}^{\mathrm{tBu}}-\mathbf{I}$

\begin{tabular}{ccc}
\hline & ${ }^{1} \mathrm{H}(\delta),{ }^{t} \mathrm{Bu} H$ & ${ }^{31} \mathrm{P}\left\{{ }^{1} \mathrm{H}\right\}(\delta)$ \\
$\mathrm{U}\left(\mathrm{Se}_{2} \mathrm{P}^{t} \mathrm{Bu}_{2}\right)_{3} \mathrm{~F}\left(\mathbf{5 S e}-\mathbf{U}^{\mathbf{t B u}}-\mathbf{F}\right)$ & -2.71 & -786.0 \\
$\mathrm{U}\left(\mathrm{Se}_{2} \mathrm{P}^{t} \mathrm{Bu}_{2}\right)_{3} \mathrm{Cl}\left(\mathbf{4} \mathbf{S e}-\mathbf{U}^{\mathbf{t B u}}-\mathbf{C l}\right)$ & -2.31 & -690.0 \\
$\mathrm{U}\left(\mathrm{Se}_{2} \mathrm{P}^{t} \mathrm{Bu}_{2}\right)_{3} \mathrm{Br}\left(\mathbf{6 S e}-\mathbf{U}^{\mathbf{t B u}}-\mathbf{B r}\right)$ & -1.39 & -652.0 \\
$\mathrm{U}\left(\mathrm{Se}_{2} \mathrm{P}^{t} \mathrm{Bu}_{2}\right)_{3} \mathrm{I}\left(\mathbf{7 S e}-\mathbf{U}^{\mathbf{t B u}}-\mathbf{I}\right)$ & -0.30 & -635.0. \\
\hline
\end{tabular}

The solid-state structure of $\mathbf{7 S e}-\mathbf{U}^{\mathbf{t B u}}-\mathbf{I}$ is shown in Figure 6. The U1-I1 bond distance is 3.1187(9) $\AA$ and is comparable to other U(IV)-I bond lengths of 3.0603(13) and 3.034(2) $\AA$ for 
$\mathrm{UI}(\mathrm{DME})\left(\mathrm{NC}\left[{ }^{t} \mathrm{Bu}\right] \mathrm{Mes}\right)_{3}$ and $\left(\mathrm{C}_{5}{ }^{t} \mathrm{Bu}_{4} \mathrm{H}\right)_{3} \mathrm{UI}$, respectively. ${ }^{64,65}$ Table 10 lists the selected bond

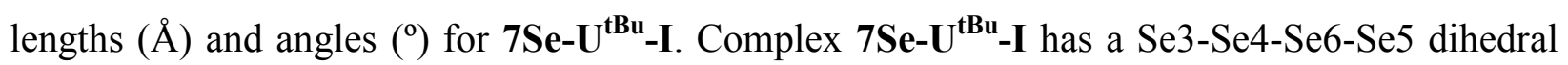
angle of $119.4^{\mathbf{o}}$ and is larger than $\mathbf{4 S e}-\mathbf{U}^{\mathbf{t B u}}-\mathbf{C l}$. The U-Se bond lengths range from 2.9312(13)2.8940(13) $\AA$ for $\mathbf{7 S e}-\mathbf{U}^{\mathrm{tBu}}-\mathbf{I}$ and are marginally shorter than $\mathbf{4 S e}-\mathbf{U}^{\mathrm{tBu}}-\mathbf{C l}$. The increase in dihedral angle of 7Se- $\mathbf{U}^{\mathrm{tBu}}-\mathbf{I}$ and shorter $\mathrm{U}$-Se bonds can be related to weaker $\pi$-donor abilities of the iodide ligand compared to those of the chloride ligand.

Table 10. Selected bond distances $(\AA)$ and angles $\left(^{(}\right)$for $\mathrm{U}\left(\mathrm{Se}_{2} \mathrm{P}^{t} \mathrm{Bu}_{2}\right)_{3} \mathrm{I}\left(\mathbf{7 S e}-\mathbf{U}^{\mathrm{tBu}}-\mathbf{I}\right)$.

\begin{tabular}{cc}
\hline U1-I1 & $3.1187(9)$ \\
U1-Se1 & $2.9038(14)$ \\
U1-Se2 & $2.9312(13)$ \\
U1-Se3 & $2.9263(13)$ \\
U1-Se4 & $2.9293(13)$ \\
U1-Se5 & $2.9198(13)$ \\
U1-Se6 & $2.8940(13)$ \\
I1-U1-Se1 & $72.15(3)$ \\
I1-U1-Se2 & $138.53(3))$ \\
Se1-U1-Se2 & $73.83(4)$ \\
Se3-U1-Se4 & $73.48(4)$ \\
Se5-U1-Se6 & $74.19(4)$ \\
Se1-P1-Se2 & $106.50(15)$ \\
Se3-P2-Se4 & $106.57(13)$ \\
Se5-P3-Se6 & $106.93(14)$ \\
\hline
\end{tabular}




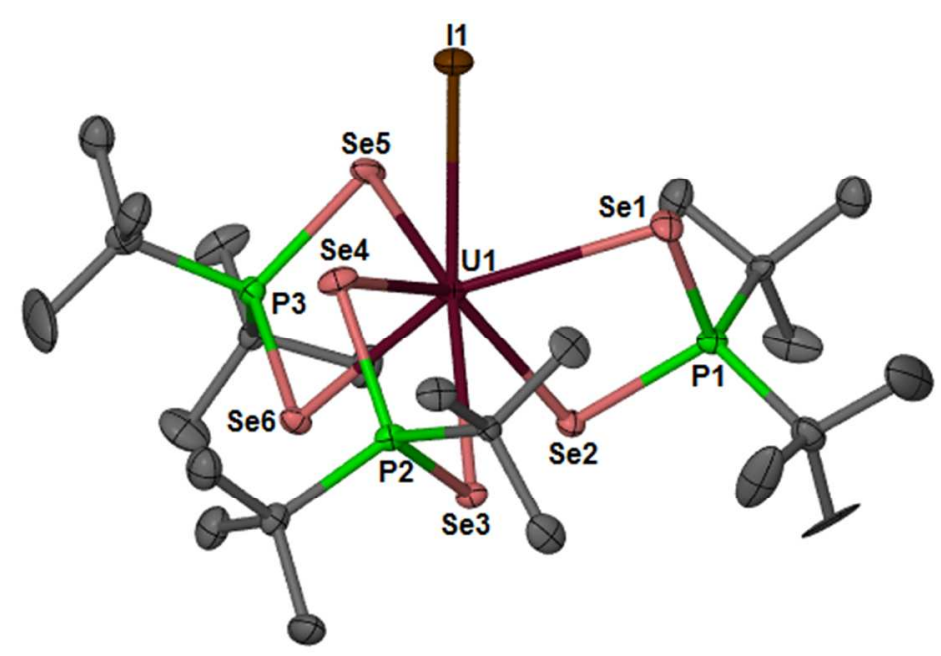

Figure 6. Thermal ellipsoid plot of $\mathrm{U}\left(\mathrm{Se}_{2} \mathrm{P}^{t} \mathrm{Bu}_{2}\right)_{3} \mathrm{I}\left(\mathbf{7 S e}-\mathbf{U}^{\mathrm{tBu}}-\mathbf{I}\right)$. Thermal ellipsoids at $50 \%$ and hydrogens omitted for clarity.

Electronic Structure Calculations. M-E bond lengths, averaged over the eight bonds in each complex, are compared in Table S5. Theoretical values are in very good agreement with experiment, with the largest difference being $0.016 \AA$ in $\mathbf{1 S e - T h}{ }^{i} \mathbf{P r}$ and many values differing by less than $0.01 \AA$. When taking the mean average deviation in bond length for a given complex into account, experimental and theoretical values overlap, justifying the choice of model chemistry.

To examine the electronic structure of these complexes, the Quantum Theory of Atoms in Molecules (QTAIM) approach was performed, in analogy with previous studies. Properties of the electron density at QTAIM derived An-E bond critical points (BCPs) are summarized in Table 11. While these properties are indicative of predominantly ionic interactions, BCP densities are higher in uranium complexes than in thorium analogues, implying greater covalency in the former. Coordination by sulfur also results in BCP densities higher than those found for metal-selenium bonds. These trends, which are mirrored by the BCP energy densities $H$, are as seen previously, ${ }^{6}$ although the difference between values obtained for uranium and 
thorium complexes is less pronounced here. Interestingly, all ${ }^{i} \operatorname{Pr}$ derivatives exhibit higher degrees of covalency than ${ }^{t} \mathrm{Bu}$ derivatives. This is commensurate with the variation in bond lengths, but is presumably due to steric rather than electronic effects.

Table 11. Topological properties at the M-E bond critical points (BCPs) of the PBE0-derived electron densities. $\rho=$ electron density, $\nabla^{2} \rho=$ Laplacian of the density, $H=$ energy density. All values are in a.u.

\begin{tabular}{|c|c|c|c|c|c|c|}
\hline Complex & \multicolumn{2}{|c|}{$\rho$} & \multicolumn{2}{c|}{$\nabla^{2} \rho$} & \multicolumn{2}{c|}{$\boldsymbol{E}=\mathrm{Ee}$} \\
\hline & $\mathrm{E}=\mathrm{S}$ & $\mathrm{E}=\mathrm{Se}$ & $\mathrm{E}=\mathrm{S}$ & $\mathrm{E}=\mathrm{Se}$ & $\mathrm{E}=\mathrm{S}$ & $\mathrm{E}=\mathrm{Se}$ \\
\hline $\mathrm{Th}\left(\mathrm{E}_{2} \mathrm{P}^{t} \mathrm{Bu}_{2}\right)_{4}$ & 0.044 & 0.040 & 0.065 & 0.050 & $-7.4 \times 10^{-3}$ & $-6.5 \times 10^{-3}$ \\
\hline $\mathrm{U}\left(\mathrm{E}_{2} \mathrm{P}^{t} \mathrm{Bu}_{2}\right)_{4}$ & 0.047 & 0.042 & 0.076 & 0.059 & $-7.9 \times 10^{-3}$ & $-6.9 \times 10^{-3}$ \\
\hline $\mathrm{Th}\left(\mathrm{E}_{2} \mathrm{P}^{i} \mathrm{Pr}_{2}\right)_{4}$ & 0.046 & 0.041 & 0.068 & 0.051 & $-8.0 \times 10^{-3}$ & $-6.8 \times 10^{-3}$ \\
\hline $\mathrm{U}\left(\mathrm{E}_{2} \mathrm{P}^{i} \mathrm{Pr}_{2}\right)_{4}$ & 0.050 & 0.043 & 0.080 & 0.060 & $-8.9 \times 10^{-3}$ & $-7.2 \times 10^{-3}$ \\
\hline
\end{tabular}

The QTAIM definition of an atom allows for the evaluation of both one- and two-electron integrated properties. The atomic charge $q$ (a one-electron property) and the localization and delocalization indices $\lambda$ and $\delta$ (two-electron properties) are summarized for $\mathrm{U}, \mathrm{Th}, \mathrm{S}$ and $\mathrm{Se}$ in Table 12. These data again support the characterization of uranium as exhibiting greater covalent character than thorium. In all complexes, delocalization indices (the number of electrons shared between two atoms), which can be considered an alternative measure of covalent character, $6,66,67$ are $\sim 0.05$ a.u. greater in uranium complexes than in the thorium analogues. It has previously been noted that the difference between atomic number $Z$ and localization index $\lambda$ correlates with oxidation state in f-element complexes ${ }^{66,67}$ and we also find this correlation here: $Z-\lambda$ values fall in the range $4.15-4.29$, close to the formal +4 oxidation state.

Table 12. Integrated QTAIM properties of the PBE0-derived electron densities. $q=$ atomic charge, $\lambda=$ localization index, $\delta=$ delocalization index. All values are in a.u.

\begin{tabular}{|c|c|c|c|c|c|c|c|c|}
\hline Complex & \multicolumn{4}{|c|}{$\mathbf{E}=\mathbf{S}$} & \multicolumn{4}{c|}{$\mathbf{E}=\mathbf{S e}$} \\
\hline & $\boldsymbol{q}(\mathbf{A n})$ & $\lambda(\mathbf{M})$ & $\boldsymbol{q}(\mathbf{E})$ & $\delta(\mathbf{A n}, \mathbf{E})$ & $\boldsymbol{q}(\mathbf{A n})$ & $\lambda(\mathbf{A n})$ & $\boldsymbol{q}(\mathbf{E})$ & $\delta(\mathbf{A n}, \mathbf{E})$ \\
\hline $\mathrm{Th}\left(\mathrm{E}_{2} \mathrm{P}^{t} \mathrm{Bu}_{2}\right)_{4}$ & +2.45 & 85.77 & -0.883 & 0.414 & +2.29 & 85.85 & -0.563 & 0.431 \\
\hline
\end{tabular}




\begin{tabular}{|c|c|c|c|c|c|c|c|c|}
\hline $\mathrm{U}\left(\mathrm{E}_{2} \mathrm{P}^{t} \mathrm{Bu}_{2}\right)_{4}$ & +2.27 & 87.74 & -0.857 & 0.461 & +2.09 & 87.82 & -0.533 & 0.481 \\
\hline $\mathrm{Th}\left(\mathrm{E}_{2} \mathrm{P}^{i} \mathrm{Pr}_{2}\right)_{4}$ & +2.45 & 85.75 & -0.923 & 0.420 & +2.29 & 85.84 & -0.577 & 0.432 \\
\hline $\mathrm{U}\left(\mathrm{E}_{2} \mathrm{P}^{i} \mathrm{Pr}_{2}\right)_{4}$ & +2.27 & 87.71 & -0.905 & 0.471 & +2.10 & 87.81 & -0.549 & 0.484 \\
\hline
\end{tabular}

In contrast to $\rho_{\mathrm{BCP}}$ values, delocalization indices suggest the An-Se bonds to be more covalent than the An-S bonds. This was previously observed in other dithiophosphinate and diselenophosphinate actinide complexes, ${ }^{6}$ and was rationalized by arguing that $\rho_{\mathrm{BCP}}$ is strongly sensitive to bond length, in which there is significant variation between the An-S and An-Se bonds. Bearing in mind the delocalization indices and previous analysis, ${ }^{6}$ we therefore conclude the An-Se bonds in these complexes to exhibit marginally more covalent character than the An-S analogues.

Overall, we have synthesized a series of dithio- and diselenophosphinate complexes of thorium(IV) and uranium(IV). The alkyl-substituents on the phosphinate ligands provide insight into the structure and bonding of Cyanex 301 as an extractor ligand. Further spectroscopic analysis is needed to verify the nature of the calculations presented; however, the nature of actinide-ligand bonding remains a fascinating and emerging field.

\section{Conclusions}

In summary, we have synthesized and characterized a series of homoleptic $\mathrm{An}\left[\mathrm{E}_{2} \mathrm{PR}_{2}\right]_{4}(\mathrm{An}=$ Th, U; E $=\mathrm{S}, \mathrm{Se} ; \mathrm{R}={ }^{i} \mathrm{Pr},{ }^{t} \mathrm{Bu}$ ) complexes spectroscopically and determined their structures using X-ray crystallography. Using the QTAIM approach, the electronic structure of these complexes showed increasing covalent bonding character in actinide-selenium bonds than in the corresponding actinide-sulfur bonds. Interestingly, the isopropyl complexes showed a higher degree of covalency than the tert-butyl substituents. For the first time, reactivity was demonstrated with these type of complexes using transmetalation reactions between $\mathrm{U}\left(\mathrm{Se}_{2} \mathrm{P}^{t} \mathrm{Bu}_{2}\right)_{4}$ and $\mathrm{Cu}^{1+}, \mathrm{ZnCl}_{2}, \mathrm{Hg}_{2} \mathrm{~F}_{2}$ or $\mathrm{HgCl}_{2}$ salts, resulting in the formation of $\mathrm{U}\left[\mathrm{Se}_{2} \mathrm{P}^{t} \mathrm{Bu}_{2}\right]_{3-}$ 
$\mathrm{X}(\mathrm{X}=\mathrm{F}, \mathrm{Cl}, \mathrm{Br}, \mathrm{I})$. The coordination chemistry of soft donor ligands with thorium and uranium is becoming an increasingly studied area and the results presented here add to the intrigue that such oxophilic metal centers can show more covalent bonding character with increasing softer donor atoms.

\section{Supporting Information}

Crystallographic details, synthesis and characterization of $\mathrm{Zn}\left(\mathrm{Se}_{2} \mathrm{P}^{t} \mathrm{Bu}_{2}\right)_{2}, \mathrm{Hg}\left(\mathrm{Se}_{2} \mathrm{P}^{t} \mathrm{Bu}_{2}\right)_{2}$, and thermal ellipsoid plots as well as tables of IR spectroscopy are available free of charge via the Internet at http://pubs.acs.org.

\section{Corresponding Authors}

Email: walenskyj@missouri.edu; a.kerridge@lancaster.ac.uk

\section{Acknowledgements}

This material is based upon work supported by the U.S. Department of Homeland Security under Grant Award Number, 2012-DN-130-NF0001-02. The views and conclusions contained in this document are those of the authors and should not be interpreted as necessarily representing the official policies, either expressed or implied, of the U.S. Department of Homeland Security. AK thanks the EPSRC for the award of a Career Acceleration Fellowship (Grant Award Number EP/J002208/1) and the NSCCS for access to the 'slater' HPC facility. We also thank Dr. Charles L. Barnes for his assistance with X-ray crystallography.

\section{References}

(1) Hudson, M. J.; Harwood, L. M.; Laventine, D. M.; Lewis, F. W. Inorg. Chem. 2013, 52, 3414.

(2) Kolarik, Z. Chem. Rev. 2008, 108, 4208. 
(3) Lewis, F. W.; Hudson, M. J.; Harwood, L. M. Synlett 2011, 2011, 2609.

(4) Peterman, D. R.; Law, J. D.; Todd, T. A.; Tillotson, R. D., Separations for the Nuclear Fuel Cycle in the $21^{\text {st }}$ Century, 2009, ACS Symposium Series, 251

(5) Jones, M. B.; Gaunt, A. J.; Gordon, J. C.; Kaltsoyannis, N.; Neu, M. P.; Scott, B. L. Chem. Sci. 2013, 4, 1189.

(6) Behrle, A. C.; Barnes, C. L.; Kaltsoyannis, N.; Walensky, J. R. Inorg. Chem. 2013, 52, 10623.

(7) Lescop, C.; Arliguie, T.; Lance, M.; Nierlich, M.; Ephritikhine, M. J. Organomet. Chem. 1999, 580, 137.

(8) Matson, E. M.; Breshears, A. T.; Kiernicki, J. J.; Newell, B. S.; Fanwick, P. E.; Shores, M. P.; Walensky, J. R.; Bart, S. C. Inorg. Chem. 2014, 53, 12977.

(9) Lam, O. P.; Franke, S. M.; Heinemann, F. W.; Meyer, K. J. Am. Chem. Soc. 2012, 134, 16877.

(10) Lane, A. C.; Vollmer, M. V.; Laber, C. H.; Melgarejo, D. Y.; Chiarella, G. M.; Fackler, J. P.; Yang, X.; Baker, G. A.; Walensky, J. R. Inorg. Chem. 2014, 53, 11357.

(11) Cantat, T.; Scott, B. L.; Kiplinger, J. L. Chem. Commun. 2010, 46, 919.

(12) Gray, D. L.; Backus, L. A.; Krug von Nidda, H.-A.; Skanthakumar, S.; Loidl, A.; Soderholm, L.; Ibers, J. A. Inorg. Chem. 2007, 46, 6992.

(13) Pinkerton, A. A.; Storey, A. E.; Zellweger, J. J. Chem. Soc., Dalton Trans. 1981, 1475.

(14) Artemev, A. V.; Malysheva, S. F.; Gusarova, N. K.; Trofimov, B. A. Synthesis 2010, 2463.

(15) APEX2 Suite, Bruker AXS Inc, 2006, Madison, WI

(16) Sheldrick, G. Acta Cryst. 2015, 71, 3.

(17) Barbour, L. J. Supramol. Chem. 2001, 1, 189.

(18) Ahlrichs, R.; Bär, M.; Häser, M.; Horn, H.; Kölmel, C. Chem. Phys. Lett. 1989, 162, 165.

(19) Adamo, C.; Barone, V. J. Chem. Phys. 1999, 110, 6158.

(20) Weigend, F.; Ahlrichs, R. Phys. Chem. Chem. Phys. 2005, 7, 3297.

(21) Cao, X.; Dolg, M. J. Mol. Struct. THEOCHEM 2004, 673, 203.

(22) Küchle, W.; Dolg, M.; Stoll, H.; Preuss, H. J. Chem. Phys. 1994, 100, 7535.

(23) Hashem, E.; Swinburne, A. N.; Schulzke, C.; Evans, R. C.; Platts, J. A.; Kerridge, A.; Natrajan, L. S.; Baker, R. J. RSC Adv. 2013, 3, 4350.

(24) Woodall, S. D.; Swinburne, A. N.; Lal Banik, N.; Kerridge, A.; Di Pietro, P.; Adam, C.; Kaden, P.; Natrajan, L. S. Chem. Commun. 2015, 51, 5402.

(25) Pantazis, D. A.; Neese, F. J. Chem. Theory Comput. 2011, 7, 677.

(26) Bader, R. F. W. Atoms in Molecules: A Quantum Theory; Oxford University Press: Oxford, 1990.

(27) AIMALL (Version 14.11.23), Keith, T. A., TK Gristmall Software, 2014, Overl. Park, KS, USA

(28) Maneeprakorn, W.; Nguyen, C. Q.; Malik, M. A.; O'Brien, P.; Raftery, J. Dalton Trans. 2009, 2103.

(29) Li, K.; Xue, D. J. Phys. Chem. A 2006, 110, 11332.

(30) Haigh, C. W. Polyhedron 1995, 14, 2871.

(31) Ren, W.; Song, H.; Zi, G.; Walter, M. D. Dalton Trans. 2012, 41, 5965.

(32) Ren, W.; Zi, G.; Walter, M. D. Organometallics 2012, 31, 672.

(33) Smiles, D. E.; Wu, G.; Hayton, T. W. Inorg. Chem. 2014, 53, 10240. 
(34) Evans, W. J.; Miller, K. A.; Ziller, J. W.; DiPasquale, A. G.; Heroux, K. J.; Rheingold, A. L. Organometallics 2007, 26, 4287.

(35) Cloke, F. G. N.; Hawkes, S. A.; Hitchcock, P. B.; Scott, P. Organometallics 1994, 13, 2895.

(36) Thomson, R. K.; Scott, B. L.; Morris, D. E.; Kiplinger, J. L. C. R. Chimie 2010, 13, 790.

(37) Villiers, C.; Thuéry, P.; Ephritikhine, M. Eur. J. Inorg. Chem. 2004, 2004, 4624.

(38) Shannon, R. D. Acta Crystallogr., Sect. A: Found. Crystallogr. 1976, 32, 751.

(39) Nguyen, C. Q.; Adeogun, A.; Afzaal, M.; Malik, M. A.; O'Brien, P. Chem. Commun. 2006, 2182.

(40) Takase, M. K.; Ziller, J. W.; Evans, W. J. Chem. Eur. J. 2011, 17, 4871.

(41) Mueller, T. J.; Ziller, J. W.; Evans, W. J. Dalton Trans. 2010, 39, 6767.

(42) Evans, W. J.; Walensky, J. R.; Ziller, J. W. Chem. Eur. J. 2009, 15, 12204.

(43) Evans, W. J.; Walensky, J. R.; Furche, F.; Ziller, J. W.; DiPasquale, A. G.; Rheingold, A. L. Inorg. Chem. 2008, 47, 10169.

(44) Evans, W. J.; Davis, B. L. Chem. Rev. 2002, 102, 2119.

(45) Jensen, M. P.; Bond, A. H.; Rickert, P. G.; Nash, K. L. J. Nucl. Sci. Technol. 2002, 39,

255.

(46) Graves, C. R.; Scott, B. L.; Morris, D. E.; Kiplinger, J. L. Organometallics 2008, 27, 3335 .

(47) Graves, C. R.; Schelter, E. J.; Cantat, T.; Scott, B. L.; Kiplinger, J. L. Organometallics 2008, 27, 5371 .

(48) Thomson, R. K.; Graves, C. R.; Scott, B. L.; Kiplinger, J. L. Eur. J. Inorg. Chem. 2009, 2009, 1451.

(49) Evans, W. J.; Walensky, J. R.; Ziller, J. W. Inorg. Chem. 2010, 49, 1743.

(50) Graves, C. R.; Scott, B. L.; Morris, D. E.; Kiplinger, J. L. J. Am. Chem. Soc. 2007, 129, 11914.

(51) Graves, C. R.; Yang, P.; Kozimor, S. A.; Vaughn, A. E.; Clark, D. L.; Conradson, S. D.; Schelter, E. J.; Scott, B. L.; Thompson, J. D.; Hay, P. J.; Morris, D. E.; Kiplinger, J. L. J. Am. Chem. Soc. 2008, 130, 5272.

(52) Graves, C. R.; Vaughn, A. E.; Schelter, E. J.; Scott, B. L.; Thompson, J. D.; Morris, D. E.; Kiplinger, J. L. Inorg. Chem. 2008, 47, 11879.

(53) Graves, C. R.; Kiplinger, J. L. Chem. Commun. 2009, 3831.

(54) Evans, W. J.; Walensky, J. R.; Ziller, J. W. Organometallics 2010, 29, 101.

(55) Montalvo, E.; Ziller, J. W.; DiPasquale, A. G.; Rheingold, A. L.; Evans, W. J. Organometallics 2010, 29, 2104.

(56) Williams, U. J.; Robinson, J. R.; Lewis, A. J.; Carroll, P. J.; Walsh, P. J.; Schelter, E. J. Inorg. Chem. 2014, 53, 27.

(57) Kosog, B.; La Pierre, H. S.; Heinemann, F. W.; Liddle, S. T.; Meyer, K. J. Am. Chem. Soc. 2012, 134, 5284.

(58) Yin, H.; Lewis, A. J.; Williams, U. J.; Carroll, P. J.; Schelter, E. J. Chem. Sci. 2013, 4, 798.

(59) Lewis, A. J.; Nakamaru-Ogiso, E.; Kikkawa, J. M.; Carroll, P. J.; Schelter, E. J. Chem. Commun. 2012, 48, 4977.

(60) King, D. M.; Tuna, F.; McInnes, E. J. L.; McMaster, J.; Lewis, W.; Blake, A. J.; Liddle, S. T. Nat. Chem. 2013, 5, 482. 
(61) Lukens, W. W.; Beshouri, S. M.; Stuart, A. L.; Andersen, R. A. Organometallics 1999, $18,1247$.

(62) Evans, W. J.; Nyce, G. W.; Johnston, M. A.; Ziller, J. W. J. Am. Chem. Soc. 2000, 122, 12019.

(63) Lukens, W. W.; Beshouri, S. M.; Blosch, L. L.; Stuart, A. L.; Andersen, R. A. Organometallics 1999, 18, 1235.

(64) Diaconescu, P. L.; Cummins, C. C. J. Am. Chem. Soc. 2002, 124, 7660.

(65) Evans, W. J.; Kozimor, S. A.; Ziller, J. W.; Fagin, A. A.; Bochkarev, M. N. Inorg. Chem. 2005, 44, 3993.

(66) Kerridge, A. Dalton Trans. 2013, 42, 16428.

(67) Kerridge, A. RSC Adv. 2014, 4, 12078. 


\section{Page 39 of 39}

1
2
3
4
5
6

$\mathrm{U}\left(\mathrm{Se}_{2} \mathrm{P}^{t} \mathrm{Bu}_{2}\right)_{4} \frac{\mathrm{HgCl}_{2}}{\mathrm{THF} / \mathrm{Toluen}}$

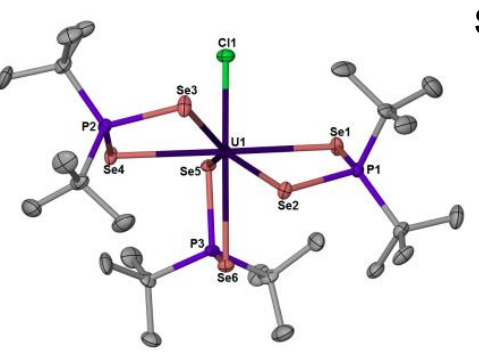

Submitted to Inorganic Chemistry

$+1 / 2$

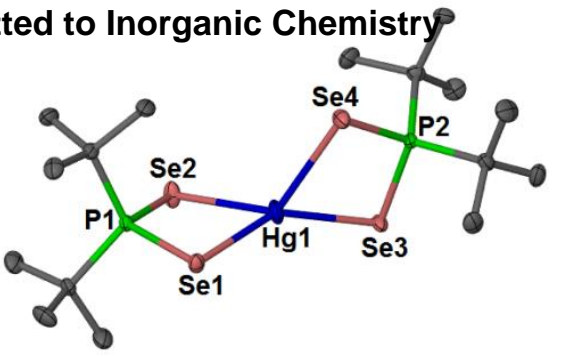

7Alkyl-substituted dithio- and diselenophosphinate complexes have been synthesized and their molecular and electronic structure examined ${ }_{9}^{8}$ using spectroscopic techniques, X-ray crystallography, and DFT calculations. Additionally, reactivity has been observed in which late 1 ffansition metal salts can be used to displace one dithio- or diselenophosphinate ligand in U(IV) complexes. 